\title{
SUPPRESSION OF CHAOS AT SLOW VARIABLES BY RAPIDLY MIXING FAST DYNAMICS THROUGH LINEAR ENERGY-PRESERVING COUPLING*
}

\author{
RAFAIL V. ABRAMOV ${ }^{\dagger}$
}

\begin{abstract}
Chaotic multiscale dynamical systems are common in many areas of science, one example being the interaction of the low-frequency dynamics in the atmosphere with the fast turbulent weather dynamics. One of the key questions about chaotic multiscale systems is how the fast dynamics affects chaos at the slow variables and, therefore, impacts uncertainty and predictability of the slow dynamics. Here we demonstrate that the linear slow-fast coupling with the total energy conservation property promotes the suppression of chaos at the slow variables through the rapid mixing at the fast variables, both theoretically and through numerical simulations. A suitable mathematical framework is developed, connecting the slow dynamics on the tangent subspaces to the infinite-time linear response of the mean state to a constant external forcing at the fast variables. Additionally, it is shown that the uncoupled dynamics for the slow variables may remain chaotic while the complete multiscale system loses chaos and becomes completely predictable at the slow variables through increasing chaos and turbulence at the fast variables. This result contradicts the common sense intuition where, naturally, one would think that coupling a slow weakly chaotic system with another much faster and much stronger mixing system would result in general increase of chaos at the slow variables.
\end{abstract}

Key words. Multiscale dynamics, chaos, mixing, predictability.

AMS subject classifications. $37 \mathrm{M}, 37 \mathrm{~N}, 60 \mathrm{G}$.

\section{Introduction}

Dynamical systems where the evolution of variables is separated between two or more different time scales are common in atmospheric/ocean science, physics, chemistry, molecular dynamics, and many other areas. The structure of these systems is typically characterized by the existence of a special subset of slow variables which evolve on a much longer time scale than the rest of the variables. In particular, one can think of the low-frequency variability models in atmospheric science, where the slow variables, usually the large scale empirical orthogonal functions describing the large-scale slowly-varying patterns in the atmosphere (such as the Arctic or North Atlantic oscillations, for example), are coupled with small-scale fast processes which are often very chaotic, turbulent, and unpredictable (with respect to the slow time scale). The inclusion of the fast processes often made the direct numerical computation of the dynamics on the slow time scale prohibitively expensive in real-world applications, which led to the development of multiscale computational methods $[12,14]$. These methods make use of the averaging formalism $[24,31,32]$ to allow for the large time discretization steps for the computation of the slow part of the dynamics. Additionally, often the dynamics on the fast time scales are not resolved, which requires suitable, often stochastic, parameterizations for the fast dynamics which are derived from the observed statistics of the fast variables (see, for example, [21-23] and references therein).

One of the key questions about the behavior of multiscale dynamics, which does not seem to be extensively addressed in the literature, is the effect of the rapidly mixing

*Received: May 16, 2011; accepted (in revised version): August 13, 2011. Communicated by David Cai.

${ }^{\dagger}$ Department of Mathematics, Statistics and Computer Science, University of Illinois at Chicago, 851 S. Morgan St., Chicago, IL 60607, USA (abramov@math.uic.edu). 
turbulent fast dynamics on the chaotic properties of the slow variables. Formally, the chaotic behavior of the whole multiscale system is determined by its largest Lyapunov characteristic exponent, which often develops at fast, strongly chaotic and rapidly mixing variables. However, the largest Lyapunov exponent of the whole system does not in practice characterize chaos at the slow variables, as it is often observed that the behavior of the slow variables can be projected far beyond the Lyapunov time scale of the fast variables. In fact, the averaging formalism in $[24,31,32]$ is based on the convergence of the slow dynamics to a limit system which does not have any fast variables, and the convergence occurs for finitely large time intervals despite the fact that the the fast dynamics become "infinitely fast" in this limit, and the corresponding Lyapunov time scale becomes infinitely short.

Here we study the chaotic behavior of slow variables by applying the averaging formalism to the dynamics on the tangent subspaces of the slow variables in a twoscale dynamical system. We demonstrate, both analytically and through numerical simulations, that if the linear coupling between the slow and fast variables preserves the total energy of the system, then the averaged dynamics systematically suppresses chaos at the slow variables. It is also speculated that the decreased ratio of the autocorrelation time scale to the advection time scale at the fast, turbulent, and rapidly mixing variables could cause this effect. Remarkably, it is observed that the full coupled system can become completely predictable and non-chaotic at the slow variables, while the uncoupled dynamics for the slow variables alone remain chaotic. The theory is also systematically extended onto the explicitly time-dependent and stochastically forced two-scale dynamics.

The manuscript is organized as follows. In Section 2 we start with a numerical experiment with an appropriately rescaled two-scale Lorenz model $[2,11,14,18]$, which demonstrates that, for only slightly affected one-point statistics such as the mean state and variance, the chaos at the slow variables is suppressed by the increasing turbulent mixing at the fast variables through the forcing parameter. In Sections 3-5 we develop a mathematical framework for the slow averaged dynamics on the tangent subspaces. For the linear energy-preserving deterministic coupling, it is shown that chaos at the slow variables is suppressed wherever the infinite-time linear response of the mean state of the fast dynamics to a constant external forcing is positive definite. It is shown that the positive-definiteness of the linear response is governed by the statistical linear stability of the fast dynamics under sufficiently small external perturbations, which, in turn, seems to be implemented by the low ratio of the autocorrelation time to the advection time scale at the fast variables. In Section 6 we go back to the Lorenz model and confirm the theoretical predictions by computing the approximate infinite-time linear response through the quasi-Gaussian fluctuation-dissipation theorem and observing how its increasing positive-definiteness correlates with suppression of chaos at the slow variables. In Section 7 we show that it is possible for the uncoupled slow dynamics to remain chaotic while the complete system with slow-fast dynamics and linear energy-preserving coupling loses chaos and becomes predictable at the slow scales by accelerating the turbulent mixing at the fast variables. In Section 8 we generalize the developed framework to include the timedependent and noisy dynamics, where the linear energy-preserving coupling may also depend on the slow time. Section 9 gives a brief exposition of what happens when the energy-preserving coupling is nonlinear in both the slow and fast variables, and has the explicit fast time dependence. In Section 10 we show that the developed framework is also applicable to the multiscale dynamics without the explicit scaling 
parameter $\varepsilon$. Section 11 summarizes the results of this work, as well as outlines the future directions of research.

\section{A remarkable behavior of the two-scale Lorenz model}

Here we consider a two-scale system of autonomous ordinary differential equations of the form

$$
\frac{\mathrm{d} \boldsymbol{x}}{\mathrm{d} t}=\boldsymbol{F}(\boldsymbol{x}, \boldsymbol{y}), \quad \frac{\mathrm{d} \boldsymbol{y}}{\mathrm{d} t}=\frac{1}{\varepsilon} \boldsymbol{G}(\boldsymbol{x}, \boldsymbol{y}),
$$

where $\boldsymbol{x}=\boldsymbol{x}(t) \in \mathbb{R}^{N_{x}}$ are the slow variables, $\boldsymbol{y}=\boldsymbol{y}(t) \in \mathbb{R}^{N_{y}}$ are the fast variables, $\boldsymbol{F}$ and $\boldsymbol{G}$ are $N_{x}$ and $N_{y}$ vector-valued functions of $\boldsymbol{x}$ and $\boldsymbol{y}$, respectively, and $\varepsilon \ll 1$ is a time-scale separation parameter.

For the two-scale system in (2.1), one can write the averaged dynamics for $\boldsymbol{x}$ alone as $\varepsilon \rightarrow 0$ using the formalism from $[2,24,31,32]$. The averaging formalism produces the averaged system for $\boldsymbol{x}$ in the form

$$
\frac{\mathrm{d} \boldsymbol{x}}{\mathrm{d} t}=\langle\boldsymbol{F}\rangle(\boldsymbol{x}), \quad\langle\boldsymbol{F}\rangle(\boldsymbol{x})=\int_{\mathcal{A}_{\boldsymbol{x}}} \boldsymbol{F}(\boldsymbol{x}, \boldsymbol{z}) \mathrm{d} \mu_{\boldsymbol{x}}(\boldsymbol{z}),
$$

where $\mu_{\boldsymbol{x}}$ is the invariant probability measure on the attractor $\mathcal{A}_{\boldsymbol{x}}$ of the limiting fast dynamics given by

$$
\frac{\mathrm{d} \boldsymbol{z}}{\mathrm{d} s}=\boldsymbol{G}(\boldsymbol{x}, \boldsymbol{z}),
$$

where $\boldsymbol{z}=\boldsymbol{z}(s)$, while $\boldsymbol{x}$ is a fixed constant parameter for (2.3) and, consequently, $\mu_{\boldsymbol{x}}$. Here we tacitly assume the ergodicity of $\mu_{\boldsymbol{x}}$, as well as that (2.2), constitutes an approximation to (2.1) for the finite times in the limit as $\varepsilon \rightarrow 0$ (for details, see [24,31, 32 ] and references therein). For the above formalism to work, the right-hand side of the averaged system in $(2.2)$ must not be $O(\varepsilon)$; otherwise, higher-order homogenization techniques are needed [31].

We choose the two-scale forced damped Lorenz model $[1,2,11,14,18]$ for the computational study of the dynamical properties of a two-scale slow-fast process with generic features of atmospheric dynamics, such as the presence of linearly unstable waves, strong nonlinearity, forcing, dissipation, chaos, and mixing. The two-scale forced damped Lorenz model is given by

$$
\begin{gathered}
\dot{x}_{i}=x_{i-1}\left(x_{i+1}-x_{i-2}\right)-x_{i}+F_{x}-\frac{\lambda_{y}}{J} \sum_{j=1}^{J} y_{i, j}, \\
\dot{y}_{i, j}=\frac{1}{\varepsilon}\left[y_{i, j+1}\left(y_{i, j-1}-y_{i, j+2}\right)-y_{i, j}+F_{y}+\lambda_{x} x_{i}\right],
\end{gathered}
$$

where $1 \leq i \leq N_{x}, 1 \leq j \leq J$. The following notations are adopted above:

- $\boldsymbol{x}$ is the set of the slow variables of size $N_{x}$. The following periodic boundary conditions hold for $\boldsymbol{x}: x_{i+N_{x}}=x_{i}$;

- $\boldsymbol{y}$ is the set of the fast variables of size $N_{y}=N_{x} J$ where $J$ is a positive integer. The following boundary conditions hold for $\boldsymbol{y}: y_{i+N_{x}, j}=y_{i, j}$ and $y_{i, j+J}=y_{i+1, j}$

- $F_{x}$ and $F_{y}$ are the constant forcing parameters; 
- $\lambda_{x}$ and $\lambda_{y}$ are the coupling parameters;

- $\varepsilon$ is the time scale separation parameter.

Originally in $[11,14,18]$ there was no constant forcing $F_{y}$ term in the equation for $\boldsymbol{y}$-variables in (2.4), however, in its absence the behavior of the $\boldsymbol{y}$-variables is strongly dissipative [1,2]. Here, as in [2], we add a constant forcing $F_{y}$ in the right-hand side of the second equation in (2.4) to induce the strongly chaotic behavior of the $\boldsymbol{y}$-variables with large positive Lyapunov exponents. It is demonstrated in [14] that the averaging formalism for $(2.4)$ is valid.

In the Lorenz model (2.4), $F_{x}$ and $F_{y}$ regulate the chaos and mixing of the $\boldsymbol{x}$ and $\boldsymbol{y}$ variables, respectively $[1-6,20]$. However, the mean state and mean energy are also affected by the changes in forcing, which affects the mean and energy trends in coupling for the fixed coupling parameters. To adjust the effect of coupling independently of forcing, here we perform the rescaling of the Lorenz model as in [20]. Consider the uncoupled Lorenz model

$$
\frac{\mathrm{d}}{\mathrm{d} t} x_{i}=x_{i-1}\left(x_{i+1}-x_{i-2}\right)-x_{i}+F
$$

with the same periodic boundary conditions as above [19]. Observe that the long term statistical mean state $\bar{x}$ and the standard deviation $\beta$ in (2.5) are the same for all $x_{i}$ due to the translational invariance. Now, we rescale $\boldsymbol{x}$ and $t$ as

$$
x_{i}=\bar{x}+\beta q_{i}, \quad t=\frac{\tau}{\beta},
$$

where the new variables $\boldsymbol{q}$ have zero mean state and unit standard deviation. In the rescaled variables, the Lorenz model becomes

$$
\frac{\mathrm{d}}{\mathrm{d} \tau} q_{i}=q_{i-1}\left(q_{i+1}-q_{i-2}\right)+\frac{1}{\beta}\left[\bar{x}\left(q_{i+1}-q_{i-2}\right)-q_{i}\right]+\frac{F-\bar{x}}{\beta^{2}},
$$

where $\bar{x}$ and $\beta$ are, of course, the functions of $F$. In addition to setting the mean state and variance of $q_{i}$ to zero and one, respectively, due to the time rescaling the autocorrelation functions of $\boldsymbol{z}$ acquire identical time scaling for any $F$ (for details, see $[20])$.

Here, we similarly rescale the two-scale Lorenz model from (2.4):

$$
\begin{aligned}
& \dot{x}_{i}=x_{i-1}\left(x_{i+1}-x_{i-2}\right)+\frac{1}{\beta_{x}}\left(\bar{x}\left(x_{i+1}-x_{i-2}\right)-x_{i}\right)+\frac{F_{x}-\bar{x}}{\beta_{x}^{2}}-\frac{\lambda_{y}}{J} \sum_{j=1}^{J} y_{i, j}, \\
& \dot{y}_{i, j}=\frac{1}{\varepsilon}\left[y_{i, j+1}\left(y_{i, j-1}-y_{i, j+2}\right)+\frac{1}{\beta_{y}}\left(\bar{y}\left(y_{i, j-1}-y_{i, j+2}\right)-y_{i, j}\right)+\frac{F_{y}-\bar{y}}{\beta_{y}^{2}}+\lambda_{x} x_{i}\right],
\end{aligned}
$$

where $\bar{x}, \bar{y}, \beta_{x}$, and $\beta_{y}$ are the long term means and variances of the corresponding uncoupled system in (2.5) with either $F_{x}$ or $F_{y}$ set as a constant forcing. Additionally, we write the fast limiting dynamics for $(2.8)$ as

$$
\dot{z}_{i, j}=z_{i, j+1}\left(z_{i, j-1}-z_{i, j+2}\right)+\frac{1}{\beta_{y}}\left(\bar{z}\left(z_{i, j-1}-z_{i, j+2}\right)-z_{i, j}\right)+\frac{F_{y}-\bar{z}}{\beta_{y}^{2}}+\lambda_{x} x_{i},
$$

where $\boldsymbol{x}$ is given as an external parameter, as in (2.3). The rescaling of the model implies that the average mean states of $\boldsymbol{x}$ and $\boldsymbol{y}$ are close to zero, and in this case the 


\begin{tabular}{|c||c|c|c|c|}
\hline \multicolumn{5}{|c|}{$N_{x}=10, N_{y}=40, F_{x}=6, \lambda_{x}=\lambda_{y}=0.25, \varepsilon=0.01$} \\
\hline$F_{y}$ & $\boldsymbol{x}$-mean & $\boldsymbol{x}$-var & $\boldsymbol{y}$-mean & $\boldsymbol{y}$-var \\
\hline \hline 6 & $9.64 \cdot 10^{-3}$ & 0.9451 & $-2.38 \cdot 10^{-3}$ & 1.066 \\
8 & $2.817 \cdot 10^{-2}$ & 0.9514 & $-1.466 \cdot 10^{-2}$ & 1.098 \\
12 & $2.05 \cdot 10^{-2}$ & 0.9336 & $-2.719 \cdot 10^{-2}$ & 1.139 \\
16 & $-1.353 \cdot 10^{-2}$ & 0.9006 & $-4.028 \cdot 10^{-2}$ & 1.153 \\
24 & $-6.972 \cdot 10^{-2}$ & 0.8434 & $-6.075 \cdot 10^{-2}$ & 1.167 \\
\hline
\end{tabular}

TABLE 2.1. The mean states and variances of the $\boldsymbol{x}$ and $\boldsymbol{y}$ variables for the rescaled Lorenz model in (2.8) with the following parameters: $N_{x}=10, N_{y}=40, F_{x}=6, F_{y}=6,8,12,16$ and 24 , $\lambda_{x}=\lambda_{y}=0.25, \varepsilon=0.01$.

slow averaged system simply becomes the rescaled Lorenz model in (2.7), where the right-hand side is $O(1)$. Therefore, the homogenization techniques from [31] are not needed, just like for the unrescaled Lorenz model in [14].

Below, we show the computed statistics of the rescaled Lorenz model (2.8) with the following parameters: $N_{x}=10, N_{y}=40, F_{x}=6, F_{y}=6,8,12,16$ and 24, $\lambda_{x}=\lambda_{y}=0.25, \varepsilon=0.01$ (the time scale separation between $\boldsymbol{x}$ and $\boldsymbol{y}$ is 100 times). The slow forcing parameter $F_{x}=6$ is chosen so that the slow dynamics are not too chaotic, mimicking the behavior of low-frequency variability in the atmosphere (it is known from the previous work $[1-6,20]$ that for $F=6$ the dynamics of the uncoupled model in (2.5) are weakly chaotic). The coupling parameters $\lambda_{x}$ and $\lambda_{y}$ are set to 0.25 so that they are neither too small, nor too large, to ensure the rich interaction between the slow and fast variables without linearizing the rescaled Lorenz system too much. The time-scale separation parameter $\varepsilon=0.01$ is also chosen so that it is neither too large, nor too small (the time scale separation by two orders of magnitude is consistent, for instance, with the difference between annual and diurnal cycles in the atmosphere). Here and everywhere else, the simple 2nd order Runge-Kutta scheme is used to integrate the model in (2.8) forward in time with the discrete time step $\Delta t=10^{-5}$ and the time averaging window of 10000 time units.

In the rescaled Lorenz model (2.8), it turns out that the values of $F_{x}$ and $F_{y}$ do not significantly affect the mean state and mean energy for both the slow variables $\boldsymbol{x}$ and fast variables $\boldsymbol{y}$. To show this, in Table 2.1 we display the mean states and variances of both $\boldsymbol{x}$ and $\boldsymbol{y}$ for the rescaled Lorenz model in (2.8). Observe that, despite different forcing regimes, the means and variances for both $\boldsymbol{x}$ and $\boldsymbol{y}$ are almost unchanged the mean states being near zero while the variances being near one as designed by the rescaling for the uncoupled model in (2.7). Here note that while the rescaling was carried out for the corresponding uncoupled model (where it sets the mean state to zero and variance to one precisely), using the same rescaling parameters in the coupled model (2.8) still sets its means and variances near prescribed values zero and one, respectively (although not precisely).

However, the different values of the fast forcing $F_{y}$ introduce some changes in the statistics (such as the probability density functions and the time autocorrelation functions) of both the slow variables $\boldsymbol{x}$ of (2.8), and the fast limiting variables $\boldsymbol{z}$ of (2.9). To show this, in Figure 2.1 we demonstrate the probability density functions and the time autocorrelation functions of both $\boldsymbol{x}$ and $\boldsymbol{z}$ (where the free parameter $\boldsymbol{x}$ is set to its mean value). Observe that in Figure 2.1 different values of the fast forcing $F_{y}$ do not significantly affect the probability density functions for both $\boldsymbol{x}$ 

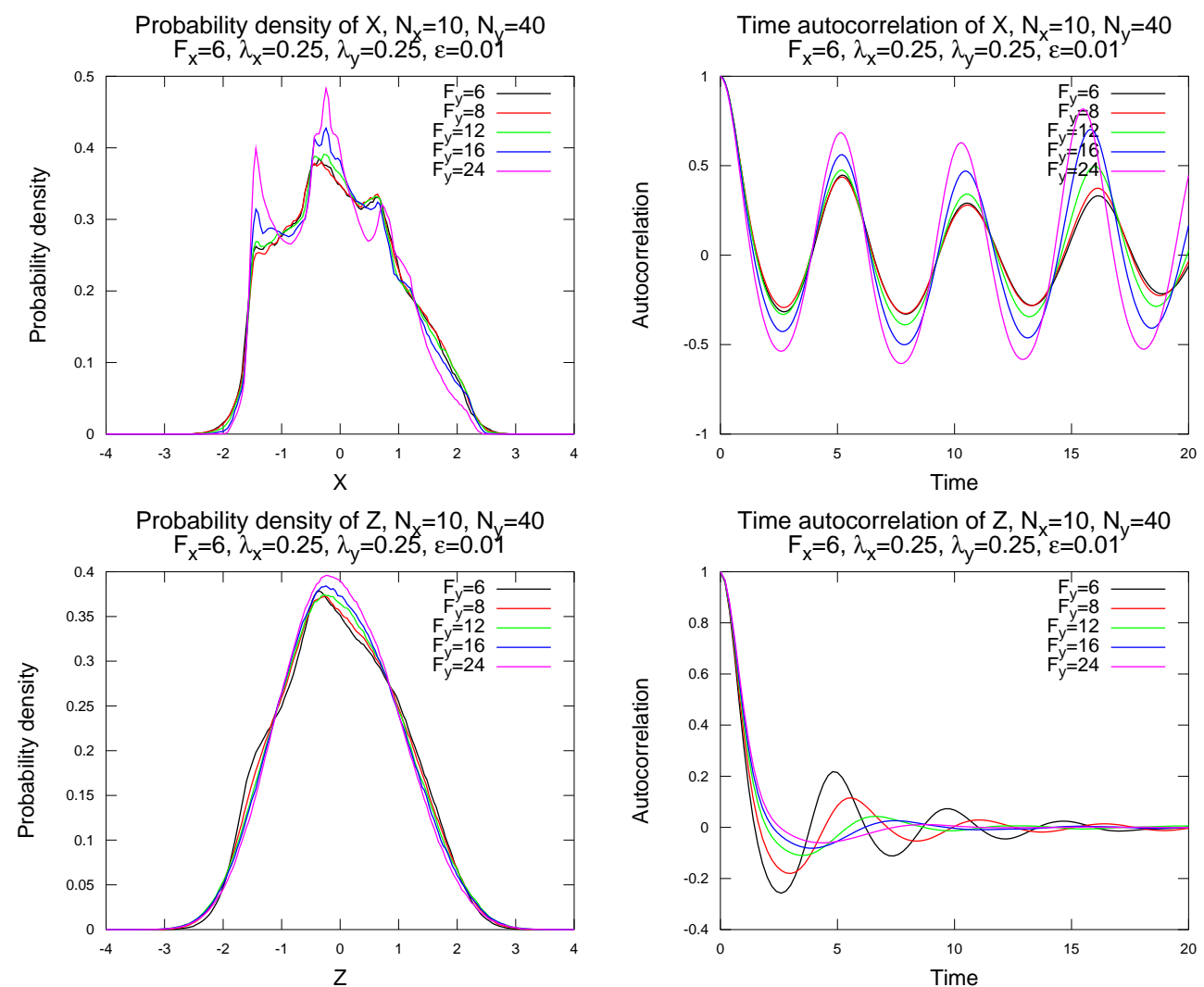

FIG. 2.1. Probability density functions and time autocorrelation functions for $\boldsymbol{x}$ of (2.8) and $\boldsymbol{z}$ of (2.9) (with free parameter $\boldsymbol{x}$ set to its mean value) with the following parameters: $N_{x}=10$, $N_{y}=40, F_{x}=6, F_{y}=6,8,12,16$ and $24, \lambda_{x}=\lambda_{y}=0.25, \varepsilon=0.01$.

and $\boldsymbol{z}$ - they are quite similar with some variations. Perhaps, the most noticeable qualitative change can be observed in the time autocorrelation functions for both $\boldsymbol{x}$ and $\boldsymbol{z}$ - for smaller values of $F_{y}$ the time autocorrelation functions of $\boldsymbol{x}$ oscillate with somewhat smaller amplitude (which indicates somewhat stronger mixing), while the time autocorrelation functions of $\boldsymbol{z}$ display visibly longer decay, which indicates weaker mixing. Having observed the statistics, we turn our attention to the chaotic behavior of the slow variables $\boldsymbol{x}$ for the same range of parameters. As the slow variables $\boldsymbol{x}$ are coupled to the fast variables $\boldsymbol{y}$, the straightforward computation of the largest Lyapunov exponent will characterize the chaos at the fast variables $\boldsymbol{y}$ and, therefore, a different method should be used to quantify chaos at the slow variables. Here we are going to observe the average divergence behavior in time between the short-time (half of the time unit) running averages $\langle\boldsymbol{x}\rangle(t)$ of the slow time series $\boldsymbol{x}(t)$, which are initially generated very closely to each other. Namely, we perform the following computation:

- We start with a generic initial condition $\left(\boldsymbol{x}_{0}, \boldsymbol{y}_{0}\right)$, and propagate it for 1000 time units to let it settle on the chaotic attractor of the rescaled Lorenz model in $(2.8)$;

- A perturbed $(\boldsymbol{x}, \boldsymbol{y})$-state is generated by a small random deviation (of order 


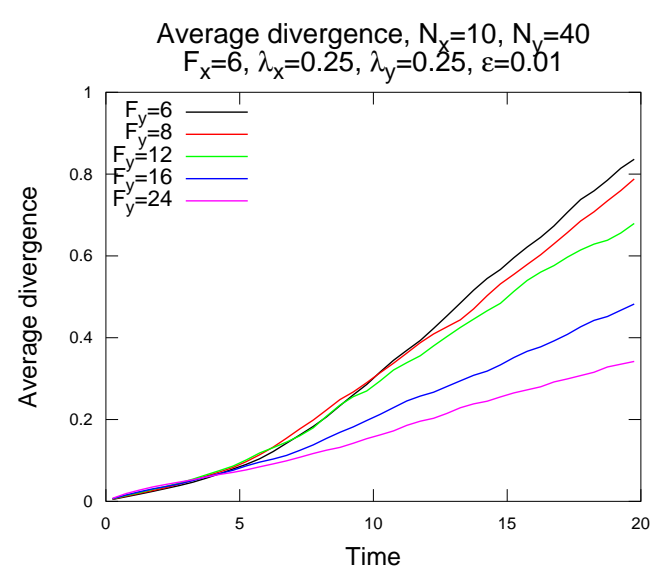

FIG. 2.2. Average divergence between perturbed and unperturbed running averages of the slow variables for the rescaled Lorenz model in (2.8) with the following parameters: $N_{x}=10, N_{y}=40$, $F_{x}=6, F_{y}=6,8,12,16$ and $24, \lambda_{x}=\lambda_{y}=0.25, \varepsilon=0.01$.

$\varepsilon)$ from the computed trajectory. Then, the original trajectory $\boldsymbol{x}(t)$ and the perturbed state $\boldsymbol{x}^{\prime}(t)$ are integrated forward in time in parallel. The time evolution of the divergence $\left\|\left\langle\boldsymbol{x}^{\prime}\right\rangle(t)-\langle\boldsymbol{x}\rangle(t)\right\|$ between the original and the perturbed trajectory is recorded;

- The latter operation is repeated 500 times with different snapshots of the same long-term trajectory, spaced by 20 time units, and averaged. The result is the time evolution of the divergence in $\langle\boldsymbol{x}\rangle(t)$ between the original and perturbed trajectory, averaged over the attractor (averaging window of 10000 time units) of the rescaled Lorenz model in (2.8).

The short time-averaging window of half of the time unit for the running average $\langle\boldsymbol{x}\rangle(t)$ ensures that the slow variables $\boldsymbol{x}(t)$ do not change much during this window, while the fast time series $\boldsymbol{y}(t)$ mix completely during the same short time averaging window (see the time scales of the time autocorrelation functions of $\boldsymbol{x}$ and $\boldsymbol{y}$ in Figure 2.1 for comparison). The results of this simulation for the same set of parameters are shown in Figure 2.2. Remarkably, the chaos in the slow $\boldsymbol{x}$-variables is consistently suppressed as the fast forcing $F_{y}$ increases, as the unperturbed and perturbed slow running averages $\langle\boldsymbol{x}\rangle(t)$ diverge from each other slower and slower in time. It cannot be caused by the changing statistical mean or variance of the slow or fast variables creating average counteracting forcing at the slow variables, as Table 2.1 clearly indicates that the mean states and variances of both the slow and fast variables do not change by a significant amount for different $F_{y}$. This chaos suppression effect at the slow variables appears to become somewhat stronger as the time-scale separation is increased: in Figure 2.3 we show the same average divergence as in Figure 2.2, but with the time-scale separation parameter $\varepsilon$ set to 0.005 and 0.001 , respectively.

In what follows we set out to explain the observed effect of the chaos suppression at the slow variables by increasing the turbulent mixing at the fast variables, without affecting the mean forcing or variability. As the effect pertains when $\varepsilon$ is decreased, one would expect the averaging formalism $[24,31,32]$ in the limit as $\varepsilon \rightarrow 0$ to provide an approximate way to study the effect from the dynamical system perspective, which we systematically develop below. 

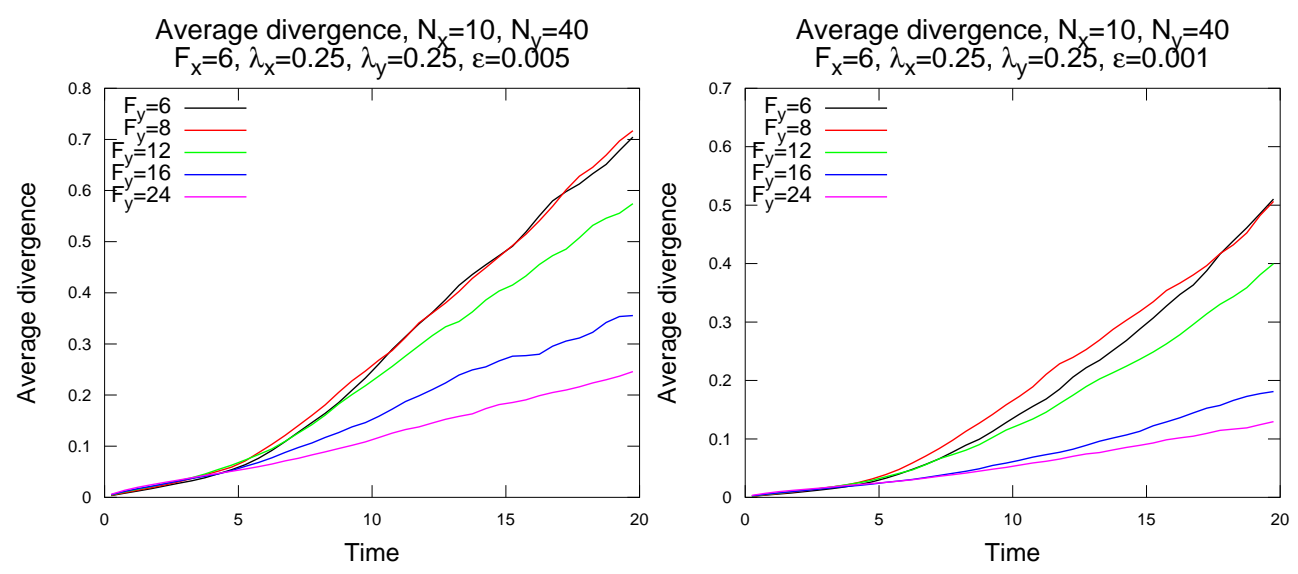

FIG. 2.3. Average divergence between perturbed and unperturbed running averages of the slow variables for the rescaled Lorenz model in (2.8) with the following parameters: $N_{x}=10, N_{y}=40$, $F_{x}=6, F_{y}=6,8,12,16$ and $24, \lambda_{x}=\lambda_{y}=0.25, \varepsilon=0.005$ and $\varepsilon=0.001$.

\section{A two-scale slow-fast system and its tangent linear model for the averaged slow variables}

To explain the observations for the two-scale rescaled Lorenz model in the previous section, here we study the behavior of the tangent linear model of the averaged dynamics in (2.2). Below, we do not attempt to rigorously prove stated claims, and instead make reasonable assumptions about the system under consideration and justify the claims under the assumptions made.

We start by deriving the tangent linear model for (2.2). Here, the necessary assumption is that the flow (or, as it is also called, the solution operator) $\boldsymbol{x}(t)=$ $\boldsymbol{X}(\boldsymbol{x}, t)$, generated by $(2.2)$, is differentiable with respect to its initial condition $\boldsymbol{x}$. In other words, we assume that the averaged tangent map

$$
\boldsymbol{T} \boldsymbol{X}_{\boldsymbol{x}}^{t}=\frac{\partial}{\partial \boldsymbol{x}} \boldsymbol{X}(\boldsymbol{x}, t)
$$

for (2.2) exists and is given by the equation

$$
\frac{\partial}{\partial t} \boldsymbol{T} \boldsymbol{X}_{\boldsymbol{x}}^{t}=\frac{\partial\langle\boldsymbol{F}\rangle}{\partial \boldsymbol{x}}(\boldsymbol{x}) \boldsymbol{T} \boldsymbol{X}_{\boldsymbol{x}}^{t}
$$

The partial derivative in (3.2) is given, formally, as

$$
\frac{\partial\langle\boldsymbol{F}\rangle}{\partial \boldsymbol{x}}(\boldsymbol{x})=\int_{\mathcal{A}_{\boldsymbol{x}}} \frac{\partial \boldsymbol{F}}{\partial \boldsymbol{x}}(\boldsymbol{x}, \boldsymbol{z}) \mathrm{d} \mu_{\boldsymbol{x}}(\boldsymbol{z})+\int_{\mathcal{A}_{\boldsymbol{x}}} \boldsymbol{F}(\boldsymbol{x}, \boldsymbol{z}) \mathrm{d} \mu_{\boldsymbol{x}}^{\prime}(\boldsymbol{z}) .
$$

Above we assume that the invariant measure $\mu_{\boldsymbol{x}}$ depends smoothly on $\boldsymbol{x}$, that is, for any $\mu_{\boldsymbol{x}}$-measurable $f(\boldsymbol{z})$ we have

$$
\begin{aligned}
& \int_{\mathcal{A}_{\boldsymbol{x}}} f(\boldsymbol{z}) \mathrm{d} \mu_{\boldsymbol{x}+\delta \boldsymbol{x}}(\boldsymbol{z})-\int_{\mathcal{A}_{\boldsymbol{x}}} f(\boldsymbol{z}) \mathrm{d} \mu_{\boldsymbol{x}}(\boldsymbol{z}) \\
= & \left(\int_{\mathcal{A}_{\boldsymbol{x}}} f(\boldsymbol{z}) \mathrm{d} \mu_{\boldsymbol{x}}^{\prime}(\boldsymbol{z})\right) \delta \boldsymbol{x}+o(\|\delta \boldsymbol{x}\|),
\end{aligned}
$$


where $\mu_{\boldsymbol{x}}^{\prime}$ denotes the derivative of $\mu_{\boldsymbol{x}}$ with respect to $\boldsymbol{x}$. The differentiability property above requires structural stability of $\mu_{\boldsymbol{x}}$ under changes in $\boldsymbol{x}$. It is known that uniformly hyperbolic diffeomorphisms on the whole $\mathbb{R}^{N_{y}}$ (Anosov) or just the nonwandering set of $\mathbb{R}^{N_{y}}$ (Axiom A) are structurally stable $[13,26-28,33]$.

Using the linear response theory $[1-3,5-7,20,25,28]$, the second term in (3.4) can be computed as the infinite-time linear response operator of (2.3) for a perturbation of the external parameter $\boldsymbol{x}$. The calculations are given in the Appendix A, and the result is

$$
\int_{\mathcal{A}_{\boldsymbol{x}}} \boldsymbol{F}(\boldsymbol{x}, \boldsymbol{z}) \mathrm{d} \mu_{\boldsymbol{x}}^{\prime}(\boldsymbol{z})=\int_{0}^{\infty} \int_{\mathcal{A}_{\boldsymbol{x}}} \frac{\partial \boldsymbol{F}}{\partial \boldsymbol{y}}\left(\boldsymbol{x}, \phi_{\boldsymbol{x}}^{s} \boldsymbol{z}\right) \boldsymbol{T}_{\boldsymbol{x}, \boldsymbol{z}}^{s} \frac{\partial \boldsymbol{G}}{\partial \boldsymbol{x}}(\boldsymbol{x}, \boldsymbol{z}) \mathrm{d} \mu_{\boldsymbol{x}}(\boldsymbol{z}) \mathrm{d} s
$$

where $\phi_{\boldsymbol{x}}^{t}$ is the flow, generated by (2.3). For convenience, we denote the left-hand side of the above formula as $\boldsymbol{H}(\boldsymbol{x})$. Thus, for the averaged tangent dynamics in (3.2) we obtain

$$
\begin{gathered}
\frac{\partial}{\partial t} \boldsymbol{T} \boldsymbol{X}_{\boldsymbol{x}}^{t}=\left(\left\langle\frac{\partial \boldsymbol{F}}{\partial \boldsymbol{x}}\right\rangle(\boldsymbol{x})+\boldsymbol{H}(\boldsymbol{x})\right) \boldsymbol{T} \boldsymbol{X}_{\boldsymbol{x}}^{t} \\
\left\langle\frac{\partial \boldsymbol{F}}{\partial \boldsymbol{x}}\right\rangle(\boldsymbol{x})=\int_{\mathcal{A}_{\boldsymbol{x}}} \frac{\partial}{\partial \boldsymbol{x}} \boldsymbol{F}(\boldsymbol{x}, \boldsymbol{z}) \mathrm{d} \mu_{\boldsymbol{x}}(\boldsymbol{z}) \\
\boldsymbol{H}(\boldsymbol{x})=\int_{0}^{\infty}\left(\int_{\mathcal{A}_{\boldsymbol{x}}} \frac{\partial \boldsymbol{F}}{\partial \boldsymbol{y}}\left(\boldsymbol{x}, \phi_{\boldsymbol{x}}^{s} \boldsymbol{z}\right) \boldsymbol{T}_{\boldsymbol{x}, \boldsymbol{z}}^{s} \frac{\partial \boldsymbol{G}}{\partial \boldsymbol{x}}(\boldsymbol{x}, \boldsymbol{z}) \mathrm{d} \mu_{\boldsymbol{x}}(\boldsymbol{z})\right) \mathrm{d} s
\end{gathered}
$$

Under the ergodicity assumption, one can replace the measure averages with the time averages:

$$
\begin{gathered}
\left\langle\frac{\partial \boldsymbol{F}}{\partial \boldsymbol{x}}\right\rangle(\boldsymbol{x})=\lim _{r \rightarrow \infty} \frac{1}{r} \int_{0}^{r} \frac{\partial}{\partial \boldsymbol{x}} \boldsymbol{F}(\boldsymbol{x}, \boldsymbol{z}(\tau)) \mathrm{d} \tau \\
\boldsymbol{H}(\boldsymbol{x})=\int_{0}^{\infty}\left(\lim _{r \rightarrow \infty} \frac{1}{r} \int_{0}^{r} \frac{\partial \boldsymbol{F}}{\partial \boldsymbol{y}}(\boldsymbol{x}, \boldsymbol{z}(\tau+s)) \boldsymbol{T}_{\boldsymbol{x}, \boldsymbol{z}(\tau)}^{s} \frac{\partial \boldsymbol{G}}{\partial \boldsymbol{x}}(\boldsymbol{x}, \boldsymbol{z}(\tau)) \mathrm{d} \tau\right) \mathrm{d} s .
\end{gathered}
$$

\section{Deterministic linear coupling with total energy conservation}

It is often the case that the deterministic parts of the coupling in $\boldsymbol{F}(\boldsymbol{x}, \boldsymbol{y})$ and $\boldsymbol{G}(\boldsymbol{x}, \boldsymbol{y})$ between the slow variables $\boldsymbol{x}$ and the fast variables $\boldsymbol{y}$ allow for total energy conservation, as long as in the absence of a random noise the uncoupled dynamics preserve the energy separately for $\boldsymbol{x}$ and $\boldsymbol{y}$. As an example, one can look at the model of mean flow - small scale interactions via topographic stress in [16], where the total energy conservation in the coupling between the zonal mean flow and small scale fluctuations is a key requirement, and topography can be viewed as a small parameter. Here we assume that the slow and fast variables are coupled through a deterministic coupling with the total energy conservation property. More precisely, we let the functions $\boldsymbol{F}(\boldsymbol{x}, \boldsymbol{y})$ and $\boldsymbol{G}(\boldsymbol{x}, \boldsymbol{y})$ take the form

$$
\boldsymbol{F}(\boldsymbol{x}, \boldsymbol{y})=\boldsymbol{f}(\boldsymbol{x})+\boldsymbol{f}^{\prime}(\boldsymbol{x}, \boldsymbol{y}), \quad \boldsymbol{G}(\boldsymbol{x}, \boldsymbol{y})=\boldsymbol{g}(\boldsymbol{y})+\boldsymbol{g}^{\prime}(\boldsymbol{x}, \boldsymbol{y}),
$$

and assume that that there exists a symmetric positive definite quadratic form

$$
E=E_{x}+\varepsilon E_{y}, \quad E_{x}=\frac{1}{2} \boldsymbol{x}^{T} \boldsymbol{S}_{x} \boldsymbol{x}, \quad E_{y}=\frac{1}{2} \boldsymbol{y}^{T} \boldsymbol{S}_{y} \boldsymbol{y}
$$


where $\boldsymbol{S}_{x}$ and $\boldsymbol{S}_{y}$ are constant symmetric positive definite matrices, which is preserved by $\boldsymbol{f}^{\prime}(\boldsymbol{x}, \boldsymbol{y})$ and $\boldsymbol{g}^{\prime}(\boldsymbol{x}, \boldsymbol{y})$ for any $\boldsymbol{x}$ and $\boldsymbol{y}$ :

$$
\boldsymbol{x}^{T} \boldsymbol{S}_{x} \boldsymbol{f}^{\prime}(\boldsymbol{x}, \boldsymbol{y})+\boldsymbol{y}^{T} \boldsymbol{S}_{y} \boldsymbol{g}^{\prime}(\boldsymbol{x}, \boldsymbol{y})=0 .
$$

For (4.3) to hold, it is sufficient to have $\boldsymbol{f}^{\prime}(\boldsymbol{x}, \boldsymbol{y})$ and $\boldsymbol{g}^{\prime}(\boldsymbol{x}, \boldsymbol{y})$ in the form

$$
\begin{gathered}
\boldsymbol{f}^{\prime}(\boldsymbol{x}, \boldsymbol{y})=\boldsymbol{S}_{x}^{-1 / 2} \boldsymbol{L}(\boldsymbol{x}, \boldsymbol{y}) \boldsymbol{S}_{y}^{1 / 2} \boldsymbol{y} \\
\boldsymbol{g}^{\prime}(\boldsymbol{x}, \boldsymbol{y})=-\boldsymbol{S}_{y}^{-1 / 2} \boldsymbol{L}^{T}(\boldsymbol{x}, \boldsymbol{y}) \boldsymbol{S}_{x}^{1 / 2} \boldsymbol{x}
\end{gathered}
$$

for an arbitrary $N_{x} \times N_{y}$ matrix-valued function $\boldsymbol{L}(\boldsymbol{x}, \boldsymbol{y})$. Additionally, if $\boldsymbol{f}^{\prime}(\boldsymbol{x}, \boldsymbol{y})$ and $\boldsymbol{g}^{\prime}(\boldsymbol{x}, \boldsymbol{y})$ are analytic on $\mathbb{R}^{N_{x}} \times \mathbb{R}^{N_{y}}$, the relations in (4.4) are also necessary: indeed, in this case $\boldsymbol{f}^{\prime}(\boldsymbol{x}, \boldsymbol{y})$ and $\boldsymbol{g}^{\prime}(\boldsymbol{x}, \boldsymbol{y})$ can be represented as

$$
\boldsymbol{f}^{\prime}(\boldsymbol{x}, \boldsymbol{y})=\boldsymbol{f}^{\prime}(\boldsymbol{x}, \mathbf{0})+\boldsymbol{f}_{1}^{\prime}(\boldsymbol{x}, \boldsymbol{y}) \boldsymbol{y}, \quad \boldsymbol{g}^{\prime}(\boldsymbol{x}, \boldsymbol{y})=\boldsymbol{g}^{\prime}(\mathbf{0}, \boldsymbol{y})+\boldsymbol{g}_{1}^{\prime}(\boldsymbol{x}, \boldsymbol{y}) \boldsymbol{x},
$$

where $\boldsymbol{f}_{1}^{\prime}(\boldsymbol{x}, \boldsymbol{y}) \boldsymbol{y}$ and $\boldsymbol{g}_{1}^{\prime}(\boldsymbol{x}, \boldsymbol{y}) \boldsymbol{x}$ represent the Taylor expansion terms above zero order in $\boldsymbol{y}$ and $\boldsymbol{x}$, respectively. As a result, (4.3) becomes

$$
\boldsymbol{x}^{T}\left[\boldsymbol{S}_{x} \boldsymbol{f}_{1}^{\prime}(\boldsymbol{x}, \boldsymbol{y})+\boldsymbol{g}_{1}^{\prime}(\boldsymbol{x}, \boldsymbol{y})^{T} \boldsymbol{S}_{y}\right] \boldsymbol{y}=-\boldsymbol{x}^{T} \boldsymbol{S}_{x} \boldsymbol{f}^{\prime}(\boldsymbol{x}, \mathbf{0})-\boldsymbol{y}^{T} \boldsymbol{S}_{y} \boldsymbol{g}^{\prime}(\mathbf{0}, \boldsymbol{y}),
$$

where setting either $\boldsymbol{x}=\mathbf{0}$ or $\boldsymbol{y}=\mathbf{0}$ turns the left-hand side to zero, which means that $\boldsymbol{f}^{\prime}(\boldsymbol{x}, \mathbf{0})=\boldsymbol{g}^{\prime}(\mathbf{0}, \boldsymbol{y})=\mathbf{0}$ for any $\boldsymbol{x}$ and $\boldsymbol{y}$, and that $\boldsymbol{f}^{\prime}(\boldsymbol{x}, \boldsymbol{y})=\boldsymbol{f}_{1}^{\prime}(\boldsymbol{x}, \boldsymbol{y}) \boldsymbol{y}, \boldsymbol{g}^{\prime}(\boldsymbol{x}, \boldsymbol{y})=$ $\boldsymbol{g}_{1}^{\prime}(\boldsymbol{x}, \boldsymbol{y}) \boldsymbol{x}$. This results in

$$
\boldsymbol{S}_{x} \boldsymbol{f}_{1}^{\prime}(\boldsymbol{x}, \boldsymbol{y})=-\boldsymbol{g}_{1}^{\prime}(\boldsymbol{x}, \boldsymbol{y})^{T} \boldsymbol{S}_{y} .
$$

Let $\boldsymbol{L}(\boldsymbol{x}, \boldsymbol{y})$ and $\boldsymbol{L}^{*}(\boldsymbol{x}, \boldsymbol{y})$ be given, respectively, by

$$
\boldsymbol{L}(\boldsymbol{x}, \boldsymbol{y})=\boldsymbol{S}_{x}^{1 / 2} \boldsymbol{f}_{1}^{\prime}(\boldsymbol{x}, \boldsymbol{y}) \boldsymbol{S}_{y}^{-1 / 2}, \quad \boldsymbol{L}^{*}(\boldsymbol{x}, \boldsymbol{y})=\boldsymbol{S}_{y}^{1 / 2} \boldsymbol{g}_{1}^{\prime}(\boldsymbol{x}, \boldsymbol{y}) \boldsymbol{S}_{x}^{-1 / 2},
$$

so that $\boldsymbol{L}^{*}(\boldsymbol{x}, \boldsymbol{y})=-\boldsymbol{L}(\boldsymbol{x}, \boldsymbol{y})^{T}$ for any $\boldsymbol{x}$ and $\boldsymbol{y}$. Therefore, we end up with

$$
\boldsymbol{f}_{1}^{\prime}(\boldsymbol{x}, \boldsymbol{y})=\boldsymbol{S}_{x}^{-1 / 2} \boldsymbol{L}(\boldsymbol{x}, \boldsymbol{y}) \boldsymbol{S}_{y}^{1 / 2}, \quad \boldsymbol{g}_{1}^{\prime}(\boldsymbol{x}, \boldsymbol{y})=-\boldsymbol{S}_{y}^{-1 / 2} \boldsymbol{L}^{T}(\boldsymbol{x}, \boldsymbol{y}) \boldsymbol{S}_{x}^{1 / 2}
$$

for some $N_{x} \times N_{y}$ matrix-valued function $\boldsymbol{L}(\boldsymbol{x}, \boldsymbol{y})$, and (4.4) follows directly.

In this work we study the simplest case of the energy-preserving coupling with $\boldsymbol{L}(\boldsymbol{x}, \boldsymbol{y})=\boldsymbol{L}$, where $\boldsymbol{L}$ is a constant matrix and does not depend on the variables $\boldsymbol{x}$ and $\boldsymbol{y}$, so that the coupling is linear with respect to $\boldsymbol{y}$ (for the slow variables) and $\boldsymbol{x}$ (for the fast variables). With (4.4), (2.1) becomes

$$
\begin{gathered}
\frac{\mathrm{d} \boldsymbol{x}}{\mathrm{d} t}=\boldsymbol{f}(\boldsymbol{x})+\boldsymbol{S}_{x}^{-1 / 2} \boldsymbol{L} \boldsymbol{S}_{y}^{1 / 2} \boldsymbol{y}, \\
\frac{\mathrm{d} \boldsymbol{y}}{\mathrm{d} t}=\frac{1}{\varepsilon}\left[\boldsymbol{g}(\boldsymbol{y})-\boldsymbol{S}_{y}^{-1 / 2} \boldsymbol{L}^{T} \boldsymbol{S}_{x}^{1 / 2} \boldsymbol{x}\right],
\end{gathered}
$$

with the limiting fast dynamics for constant $\boldsymbol{x}$ given by

$$
\frac{\mathrm{d} \boldsymbol{z}}{\mathrm{d} t}=\boldsymbol{g}(\boldsymbol{z})-\boldsymbol{S}_{y}^{-1 / 2} \boldsymbol{L}^{T} \boldsymbol{S}_{x}^{1 / 2} \boldsymbol{x}
$$


With the above changes, the integral of the time autocorrelation function in (3.6c) can be written as

$$
\boldsymbol{H}(\boldsymbol{x})=-\boldsymbol{S}_{x}^{-1 / 2} \boldsymbol{L} \boldsymbol{S}_{y}^{1 / 2}\left(\int_{0}^{\infty} \int_{\mathcal{A}_{\boldsymbol{x}}} \boldsymbol{T}_{\boldsymbol{x}, \boldsymbol{z}}^{s} \mathrm{~d} \mu_{\boldsymbol{x}}(\boldsymbol{z}) \mathrm{d} s\right) \boldsymbol{S}_{y}^{-1 / 2} \boldsymbol{L}^{T} \boldsymbol{S}_{x}^{1 / 2} .
$$

The dynamics for the averaged slow tangent map from (3.6) is now written as

$$
\frac{\partial}{\partial t} \boldsymbol{T} \boldsymbol{X}_{\boldsymbol{x}}^{t}=\left(\frac{\partial}{\partial \boldsymbol{x}} \boldsymbol{f}(\boldsymbol{x}(t))+\boldsymbol{H}(\boldsymbol{x}(t))\right) \boldsymbol{T} \boldsymbol{X}_{\boldsymbol{x}}^{t}
$$

Observe that if the slow system in (4.10a) is completely decoupled from the fast variables (for example, $\boldsymbol{L}=0$ ), then the linear tangent model above loses the term $\boldsymbol{H}(\boldsymbol{x})$, and otherwise remain the same. We study the effect of $\boldsymbol{H}(\boldsymbol{x})$ on the dynamics of (4.13) below. Observe that $\boldsymbol{H}(\boldsymbol{x})$ can be written in the form

$$
\begin{gathered}
\boldsymbol{H}(\boldsymbol{x})=-\boldsymbol{S}_{x}^{-1 / 2} \boldsymbol{L} \boldsymbol{S}_{y}^{1 / 2} \mathcal{C}(\boldsymbol{x}) \boldsymbol{S}_{y}^{-1 / 2} \boldsymbol{L}^{T} \boldsymbol{S}_{x}^{1 / 2} \\
\mathcal{C}(\boldsymbol{x})=\int_{0}^{\infty} \boldsymbol{C}(\boldsymbol{x}, s) \mathrm{d} s \\
\boldsymbol{C}(\boldsymbol{x}, s)=\int_{\mathcal{A}_{\boldsymbol{x}}} \boldsymbol{T}_{\boldsymbol{x}, \boldsymbol{z}}^{s} \mathrm{~d} \mu_{\boldsymbol{x}}(\boldsymbol{z})=\lim _{r \rightarrow \infty} \frac{1}{r} \int_{0}^{r} \boldsymbol{T}_{\boldsymbol{x}, \boldsymbol{z}(\tau)}^{s} \mathrm{~d} \tau
\end{gathered}
$$

(the last equality is obtained under the ergodicity assumption), where $\mathcal{C}(\boldsymbol{x})$ is the infinite time linear response operator of the mean state of (4.11) to a constant external forcing, with given parameter $\boldsymbol{x}$ (for details, see Appendix B). Replacing $\hat{\boldsymbol{x}}=\boldsymbol{S}_{x}^{1 / 2} \boldsymbol{x}$ and $\hat{\boldsymbol{y}}=\boldsymbol{S}_{y}^{1 / 2} \boldsymbol{y}$, which are canonical variables for the energy in (4.2) (that is, the quadratic form in (4.2) is diagonal in the new variables $\hat{\boldsymbol{x}}$ and $\hat{\boldsymbol{y}}$ ), in the new variables we obtain

$$
\hat{\boldsymbol{H}}(\boldsymbol{x})=-\boldsymbol{L} \hat{\mathcal{C}}(\boldsymbol{x}) \boldsymbol{L}^{T}
$$

Here,

$$
\hat{\boldsymbol{H}}(\boldsymbol{x})=\boldsymbol{S}_{x}^{1 / 2} \boldsymbol{H}(\boldsymbol{x}) \boldsymbol{S}_{x}^{-1 / 2}
$$

is the term $\boldsymbol{H}(\boldsymbol{x})$ in the canonical energy coordinates, while $\hat{\mathcal{C}}(\boldsymbol{x})$ is the infinite-time mean state linear response operator for (4.11) in the canonical energy coordinates $\hat{\boldsymbol{x}}$ and $\hat{\boldsymbol{y}}$ :

$$
\hat{\mathcal{C}}(\boldsymbol{x})=\boldsymbol{S}_{y}^{1 / 2} \mathcal{C}(\boldsymbol{x}) \boldsymbol{S}_{y}^{-1 / 2}
$$

since for $\hat{\boldsymbol{z}}=\boldsymbol{S}_{y}^{1 / 2} \boldsymbol{z}$ we have

$$
\hat{\boldsymbol{T}}_{\boldsymbol{x}, \hat{\boldsymbol{z}}}^{s}=\boldsymbol{S}_{y}^{1 / 2} \boldsymbol{T}_{\boldsymbol{x}, \boldsymbol{z}}^{s} \boldsymbol{S}_{y}^{-1 / 2}
$$

5. Suppression of chaos at slow variables by the linear energypreserving coupling for the rapidly decorrelating fast dynamics

Without loss of generality, we further assume that $\boldsymbol{x}$ and $\boldsymbol{y}$ are already the canonical energy variables $\hat{\boldsymbol{x}}$ and $\hat{\boldsymbol{y}}$, as if the dynamical system in (4.10) was formulated in the canonical energy variables from the beginning, such that $\boldsymbol{S}_{x}$ and $\boldsymbol{S}_{y}$ in (4.2) are 
multiples of an identity matrix. With this assumption, the term $\boldsymbol{H}(\boldsymbol{x})$ from (4.12) is written as

$$
\boldsymbol{H}(\boldsymbol{x})=-\boldsymbol{L C}(\boldsymbol{x}) \boldsymbol{L}^{T},
$$

where $\mathcal{C}(\boldsymbol{x})$ is given by (4.14b) and (4.14c), and computed from the fast limiting dynamical system given by

$$
\frac{\mathrm{d} \boldsymbol{z}}{\mathrm{d} t}=\boldsymbol{g}(\boldsymbol{z})-\boldsymbol{L}^{T} \boldsymbol{x}
$$

with given parameter $\boldsymbol{x}$.

At this point, observe that if $\boldsymbol{H}$ is negative definite, then it has a damping effect on the growth of $\boldsymbol{T} \boldsymbol{X}_{\boldsymbol{x}}^{t}$ in (4.13) (more generally, in the non-canonical variables $\boldsymbol{H}$ reduces the slow energy $E_{x}$, promoting the Lyapunov stability). However, from (5.1) we see that $\boldsymbol{H}$ can only be negative definite whenever $\mathcal{C}$ is positive definite. Indeed, let $\boldsymbol{v}^{T} \boldsymbol{H} \boldsymbol{v}<0$ for any nonzero $\boldsymbol{v}$. Then, denoting $\boldsymbol{w}=\boldsymbol{L}^{T} \boldsymbol{v}$, we obtain $\boldsymbol{w}^{T} \mathcal{C} \boldsymbol{w}>0$. Below we establish the physical conditions which promote the positive-definiteness of $\mathcal{C}(\boldsymbol{x})$.

First, observe that $\mathcal{C}(\boldsymbol{x})$ is the infinite-time linear response operator for (5.2) perturbed by a small constant forcing. In particular, if (5.2) is an Ornstein-Uhlenbeck process [30] of the form

$$
\mathrm{d} \boldsymbol{z}=\left(\boldsymbol{h}-\boldsymbol{\Gamma} \boldsymbol{z}-\boldsymbol{L}^{T} \boldsymbol{x}\right) \mathrm{d} s+\boldsymbol{\sigma} \mathrm{d} \boldsymbol{W}_{s},
$$

with $\boldsymbol{\Gamma}$ being a positive-definite matrix to (almost) ensure boundedness of the solution, then we obtain explicitly

$$
\begin{gathered}
\boldsymbol{C}(\boldsymbol{x}, s)=\boldsymbol{C}(s)=e^{-s \boldsymbol{\Gamma}} \\
\mathcal{C}(\boldsymbol{x})=\mathcal{C}=\int_{0}^{\infty} \boldsymbol{C}(s) \mathrm{d} s=\boldsymbol{\Gamma}^{-1} \\
\boldsymbol{v}^{T} \boldsymbol{H} \boldsymbol{v}=-\boldsymbol{v}^{T} \boldsymbol{L} \boldsymbol{\Gamma}^{-1} \boldsymbol{L}^{T} \boldsymbol{v}<0,
\end{gathered}
$$

which means that the term $\boldsymbol{H}$ automatically contributes towards the reduction of chaos at the slow variables whenever the fast dynamics are modeled by an OrnsteinUhlenbeck process. In general, since

$$
\langle\delta \boldsymbol{z}\rangle=\mathcal{C} \cdot \delta \boldsymbol{f}
$$

where $\delta \boldsymbol{f}$ is a small constant perturbation, and $\langle\delta \boldsymbol{z}\rangle$ is the infinite-time linear response of the mean state $\langle\boldsymbol{z}\rangle$ of (5.2), the positive-definiteness of $\mathcal{C}$ means that

$$
\langle\delta \boldsymbol{z}\rangle \cdot \delta \boldsymbol{f}>0 \text { for all sufficiently small } \delta \boldsymbol{f},
$$

that is, the response of the mean state never develops against the perturbation. It is not difficult to show that the following identity holds whenever $\delta \boldsymbol{f}$ vanishes:

$$
\frac{\mathrm{d}}{\mathrm{d} t}\langle\delta \boldsymbol{z}\rangle=-\delta \boldsymbol{f}
$$


that is, the time derivative of $\langle\delta \boldsymbol{z}\rangle$ at the moment the small constant perturbation $\delta \boldsymbol{f}$ vanishes equals this perturbation with the opposite sign. Therefore, the positivedefiniteness of $\mathcal{C}$ is equivalent to the following mean stability property of (5.2):

$$
\frac{\mathrm{d}}{\mathrm{d} t}\|\langle\delta \boldsymbol{z}\rangle\|<0
$$

that is, any sufficiently small infinite-time perturbation of the mean state $\langle\delta \boldsymbol{z}\rangle$ decreases in time at the moment when the external perturbation $\delta \boldsymbol{f}$ is removed. At present, it is not precisely clear to the author how to ascertain this property for general nonlinear $\boldsymbol{g}$ in (5.2), however, one might expect such properties to be common in strongly chaotic and mixing turbulent dynamics, as their statistical properties are often modeled by an appropriate Ornstein-Uhlenbeck process (see [21-23] and references therein), and, as shown above, the Ornstein-Uhlenbeck process satisfies this property automatically. For general nonlinear dynamics, the mean stability property must be associated with the situation where the typical Poincaré recurrence time of nonlinear motion around the mean state in (5.2) (which can be viewed as an advective time scale) is not much shorter than the turbulent mixing autocorrelation time. The reason is that, since $\boldsymbol{T}_{\boldsymbol{z}}^{0}=\boldsymbol{I}$ (the identity matrix) for any $\boldsymbol{z}$, then there always exists $a^{*}$ such that

$$
\mathcal{C}_{a}(\boldsymbol{x})=\int_{0}^{a^{*}} \boldsymbol{C}(\boldsymbol{x}, s) \mathrm{d} s \text { is positive definite for all } a, \quad 0<a<a^{*} .
$$

The positive-definiteness of $\mathcal{C}_{a}(\boldsymbol{x})$ for larger $a$ can be violated by the domination of the rotation part in $\boldsymbol{C}(\boldsymbol{x}, s)$ for larger $s$, which evolves on the advective time scale of (5.2). However, this effect can be prevented by a rapid decay of $\|\boldsymbol{C}(\boldsymbol{x}, s)\|$ for large $s$, which is governed by the turbulent mixing autocorrelation time. Thus, in general, one can expect the positive-definiteness of $\mathcal{C}(\boldsymbol{x})$ in the situations where the turbulent mixing autocorrelation time scale is not much longer than the advective time scale.

\section{Revisiting the rescaled Lorenz model}

Here, after developing the theory for the suppression of chaos at the slow variables by the rapidly mixing fast dynamics, we return back to the rescaled Lorenz model in (2.8). At this point, we can observe that the rescaled Lorenz model in (2.8) conforms to the requirements of the theory developed above, with the following properties:

- The $\boldsymbol{x}$ and $\boldsymbol{y}$ variables in the rescaled Lorenz model are already the canonical energy variables (up to constant positive factors). Indeed, set all constant and linear terms in the rescaled Lorenz model in (2.8) to zero. Then, it is easy to see that the nonlinear parts in $\boldsymbol{x}$ and $\boldsymbol{y}$ separately preserve the energies

$$
E_{x}=\frac{\lambda_{x}}{2} \sum_{i} x_{i}^{2}, \quad E_{y}=\frac{\lambda_{y}}{2 J} \sum_{i=1}^{N_{x}} \sum_{j=1}^{J} y_{i j}^{2},
$$

while the full rescaled Lorenz model without forcing and dissipation preserves the total energy of the form

$$
E=\frac{\lambda_{x}}{2} \sum_{i=1}^{N_{x}} x_{i}^{2}+\frac{\varepsilon \lambda_{y}}{2 J} \sum_{i=1}^{N_{x}} \sum_{j=1}^{J} y_{i j}^{2}=E_{x}+\varepsilon E_{y},
$$

Note that $\boldsymbol{x}$ and $\boldsymbol{y}$ in the rescaled Lorenz model are already the canonical energy variables, as the quadratic form above is diagonal. 
- The deterministic linear energy-preserving coupling is given by

$$
(\boldsymbol{L} \boldsymbol{y})_{i}=-\sum_{j=1}^{J} y_{i j}, \quad\left(\boldsymbol{L}^{T} \boldsymbol{x}\right)_{i j}=-x_{i},
$$

and $\boldsymbol{H}(\boldsymbol{x})$ is given by

$$
\boldsymbol{H}(\boldsymbol{x})=-\frac{\lambda_{x} \lambda_{y}}{J} \boldsymbol{L C}(\boldsymbol{x}) \boldsymbol{L}^{T}
$$

- The rescaled Lorenz model can be written as

$$
\begin{gathered}
\frac{\mathrm{d} \boldsymbol{x}}{\mathrm{d} t}=\boldsymbol{f}(\boldsymbol{x})+\frac{\lambda_{y}}{J} \boldsymbol{L} \boldsymbol{y}, \\
\frac{\mathrm{d} \boldsymbol{y}}{\mathrm{d} t}=\frac{1}{\varepsilon}\left[\boldsymbol{g}(\boldsymbol{y})-\lambda_{x} \boldsymbol{L}^{T} \boldsymbol{x}\right],
\end{gathered}
$$

with $\boldsymbol{f}(\boldsymbol{x})$ and $\boldsymbol{g}(\boldsymbol{y})$ given by

$$
\begin{gathered}
f_{i}(\boldsymbol{x})=x_{i-1}\left(x_{i+1}-x_{i-2}\right)+\frac{1}{\beta_{x}}\left(\bar{x}\left(x_{i+1}-x_{i-2}\right)-x_{i}\right)+\frac{F_{x}-\bar{x}}{\beta_{x}^{2}} \\
g_{i j}(\boldsymbol{y})=y_{i, j+1}\left(y_{i, j-1}-y_{i, j+2}\right)+\frac{1}{\beta_{y}}\left(\bar{y}\left(y_{i, j-1}-y_{i, j+2}\right)-y_{i, j}\right)+\frac{F_{y}-\bar{y}}{\beta_{y}^{2}}
\end{gathered}
$$

respectively.

- The slow tangent model dynamics for the rescaled Lorenz model can be written as

$$
\frac{\mathrm{d}}{\mathrm{d} t} \boldsymbol{T} \boldsymbol{X}_{\boldsymbol{x}}^{t}=\left[\frac{\partial \boldsymbol{f}}{\partial \boldsymbol{x}}(\boldsymbol{x}(t))+\boldsymbol{H}(\boldsymbol{x}(t))\right] \boldsymbol{T} \boldsymbol{X}_{\boldsymbol{x}}^{t}
$$

Overall, it turns out that in the slow-time tangent linear model for the rescaled Lorenz system in (2.8), the additional term $\boldsymbol{H}(\boldsymbol{x})$ suppresses chaos at the slow variables if the infinite-time linear response operator $\mathcal{C}(\boldsymbol{x})$ of the limiting fast dynamics, given by $(4.14 \mathrm{~b})$ and $(4.14 \mathrm{c})$, is positive definite. It is, of course, not practically feasible to compute $\boldsymbol{C}(\boldsymbol{x}, s)$ directly for all $\boldsymbol{x}$ and $s$. Here, however, we will use a suitable approximation for $\boldsymbol{C}(\boldsymbol{x}, s)$ in the form of the quasi-Gaussian linear response operator $[1-3,5-8,20]$. Namely, we approximate the average above by the average with respect to the Gaussian distribution $p_{\boldsymbol{x}}^{G}(\boldsymbol{z})$ with the same mean state and covariance matrix as $\mu_{\boldsymbol{x}}[5-7]$ :

$$
\begin{gathered}
\boldsymbol{C}(\boldsymbol{x}, s) \approx \boldsymbol{C}_{G}(\boldsymbol{x}, s)=\int_{\mathbb{R}^{N_{y}}} \boldsymbol{T}_{\boldsymbol{x}, \boldsymbol{z}}^{s} p_{\boldsymbol{x}}^{G}(\boldsymbol{z}) \mathrm{d} \boldsymbol{z}, \\
p_{\boldsymbol{x}}^{G}(\boldsymbol{z})=\exp \left(-\frac{1}{2}(\boldsymbol{z}-\overline{\boldsymbol{z}}(\boldsymbol{x}))^{T} \boldsymbol{\Sigma}^{-1}(\boldsymbol{x})(\boldsymbol{z}-\overline{\boldsymbol{z}}(\boldsymbol{x}))\right),
\end{gathered}
$$

where $\overline{\boldsymbol{z}}(\boldsymbol{x})$ and $\boldsymbol{\Sigma}(\boldsymbol{x})$ are the mean state and covariance matrix, respectively, of the fast limiting dynamics in (5.2) for fixed $\boldsymbol{x}$. Then, via integration by parts, one can rewrite $(6.9 \mathrm{a})$ as

$$
\boldsymbol{C}_{G}(\boldsymbol{x}, s)=\left[\int_{\mathbb{R}^{N_{y}}} \boldsymbol{z}(s)(\boldsymbol{z}-\overline{\boldsymbol{z}}(\boldsymbol{x}))^{T} p_{\boldsymbol{x}}^{G}(\boldsymbol{z}) \mathrm{d} \boldsymbol{z}\right] \boldsymbol{\Sigma}^{-1}(\boldsymbol{x}),
$$




\begin{tabular}{|c|c|c|c|c|}
\hline \multicolumn{5}{|c|}{$N_{x}=20, N_{y}=80, F_{x}=6, \lambda_{x}=\lambda_{y}=0.35, \varepsilon=0.01$} \\
\hline$F_{y}$ & $\boldsymbol{x}$-mean & $\boldsymbol{x}$-var & $\boldsymbol{y}$-mean & $\boldsymbol{y}$-var \\
\hline \hline 6 & $6.216 \cdot 10^{-3}$ & 0.8878 & $-9.982 \cdot 10^{-3}$ & 1.119 \\
8 & $2.34 \cdot 10^{-2}$ & 0.8728 & $-2.791 \cdot 10^{-2}$ & 1.19 \\
12 & -0.1363 & 0.6927 & $-5.553 \cdot 10^{-2}$ & 1.168 \\
16 & -0.1444 & 0.6703 & $-8.669 \cdot 10^{-2}$ & 1.199 \\
\hline
\end{tabular}

TABLE 6.1. The mean states and variances of the $\boldsymbol{x}$ and $\boldsymbol{y}$ variables for the rescaled Lorenz model in (2.8) with the following parameters: $N_{x}=20, N_{y}=80, F_{x}=6, F_{y}=6,8,12$ and $16, \lambda_{x}=\lambda_{y}=0.35$, $\varepsilon=0.01$.

which after going back to the time averages becomes the time autocorrelation function of the form

$$
\boldsymbol{C}_{G}(\boldsymbol{x}, s)=\left[\lim _{r \rightarrow \infty} \frac{1}{r} \int_{0}^{r} \boldsymbol{z}(\tau+s)(\boldsymbol{z}(\tau)-\overline{\boldsymbol{z}}(\boldsymbol{x}))^{T} \mathrm{~d} \tau\right] \boldsymbol{\Sigma}^{-1}(\boldsymbol{x}),
$$

which, after denoting $\boldsymbol{z}(t)=\overline{\boldsymbol{z}}(\boldsymbol{x})+\boldsymbol{z}^{\prime}(t)$, can be written in the form

$$
\boldsymbol{C}_{G}(\boldsymbol{x}, s)=\left[\lim _{r \rightarrow \infty} \frac{1}{r} \int_{0}^{r} \boldsymbol{z}^{\prime}(\tau+s) \boldsymbol{z}^{T}(\tau) \mathrm{d} \tau\right] \boldsymbol{\Sigma}^{-1}(\boldsymbol{x})
$$

Even after the above simplification, it is still not practically feasible to compute $\boldsymbol{C}(\boldsymbol{x}, s)$ at many points $\boldsymbol{x}$, as needed in (4.13). Here, however, we only need to observe $\boldsymbol{C}(\boldsymbol{x}, s)$ and $\mathcal{C}(\boldsymbol{x})$ for diagnostic purposes, to relate to the trends in Figures 2.2 and 2.3. Thus, we compute $\mathcal{C}_{G}(\boldsymbol{x})$ at the single point $\boldsymbol{x}=\overline{\boldsymbol{x}}$, which is the longterm mean state of the slow variables in (2.8). Since the motion of the slow variables occurs in the vicinity of their mean state, the computed $\mathcal{C}_{G}(\overline{\boldsymbol{x}})$ should generally reflect the trends happening around that point. In Figure 6.1 we show the computed operators $\mathcal{C}_{G}(\overline{\boldsymbol{x}})$, together with the smallest eigenvalues of the symmetric parts of those operators (as skew-symmetric parts do not contribute to positive-definiteness). Obviously, the effect of chaos suppression in (6.8) is stronger with increasing smallest eigenvalue of $\mathcal{C}(\boldsymbol{x})$ in (6.4), for which $\mathcal{C}_{G}(\overline{\boldsymbol{x}})$ is a rough estimate. Indeed, observe that the smallest eigenvalue of the response systematically increases with increased $F_{y}$ (in fact, for $F_{y}=6$ the operator is not even positive definite). Of course, these results are no more than crude estimates of the actual trends, as in reality the response operators must be computed for each $\boldsymbol{x}$ and using the exact formulas in (4.14b) and (4.14c), rather than the one-point estimate under Gaussian assumption. Yet, as one can see, even these crude approximations help to connect the trends in Figure 6.1 with the surprising behavior displayed in Figures 2.2 and 2.3 in Section 2.

\footnotetext{
7. Complete suppression of chaos at slow variables while the uncoupled slow dynamics remain chaotic

One of the key questions in atmosphere/ocean science is whether the uncoupled system, consisting of slow variables only, is more or less chaotic than its original version, coupled with the fast, often unresolved or underresolved variables. Indeed, often scientists work with uncoupled models consisting of slow variables only, where coupling terms were replaced with the estimates of the long-term averages of the corresponding fast variables (for example, the T21 barotropic model with the realistic Earth topography $[7,15,29]$ ), and study dynamical properties of the slow variables in the uncoupled models. The common sense in this case suggests that if the uncoupled
} 

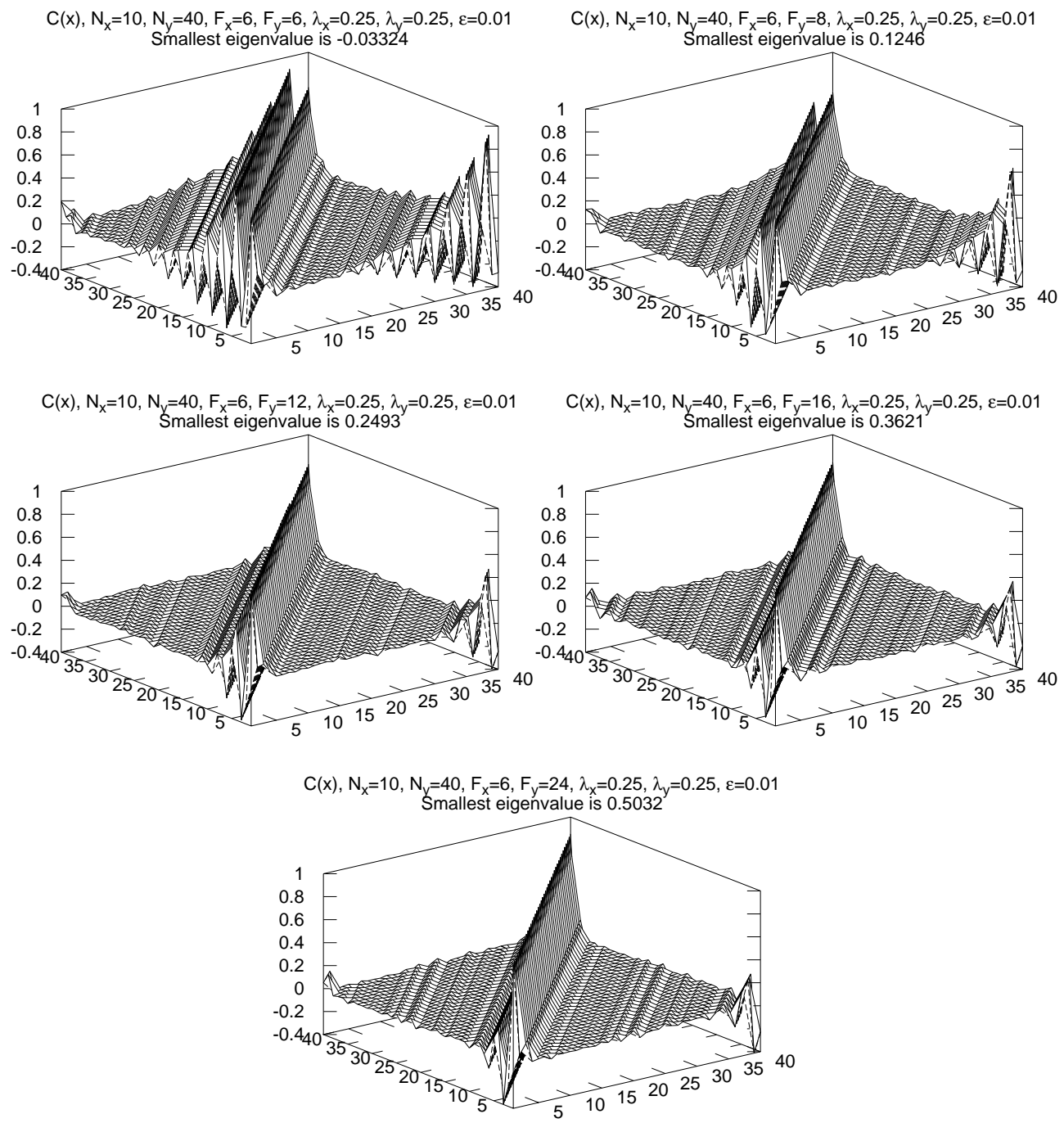

FIG. 6.1. Quasi-Gaussian approximations of averaged infinite-time linear response operators $\mathcal{C}_{G}(\overline{\boldsymbol{x}})$ for the rescaled Lorenz model in (2.8) with the following parameters: $N_{x}=10, N_{y}=40$, $F_{x}=6, F_{y}=6,8,12,16$ and $24, \lambda_{x}=\lambda_{y}=0.25, \varepsilon=0.01$.

slow model is chaotic, then, naturally, its original version coupled with fast rapidly mixing dynamics should be even more chaotic.

Remarkably, the common sense logic in this situation is deceiving. In fact, it turns out to be possible even to reach the transition from the chaotic to stable slow dynamics by increasing the turbulent mixing at the fast variables, while the uncoupled slow dynamics remain chaotic. This generally follows from the fact that in (4.13) the term $\boldsymbol{H}$ is absent from the uncoupled dynamics, and only the Jacobian of $\boldsymbol{f}$ remains. Since $\boldsymbol{H}$ acts as a dissipation in (4.13) for positive-definite infinite-time linear response of the fast variables, it is quite natural that in its absence the uncoupled dynamics is more chaotic than the fully coupled dynamics at the slow variables.

Here we demonstrate such an example for the rescaled Lorenz model in (2.8) with 


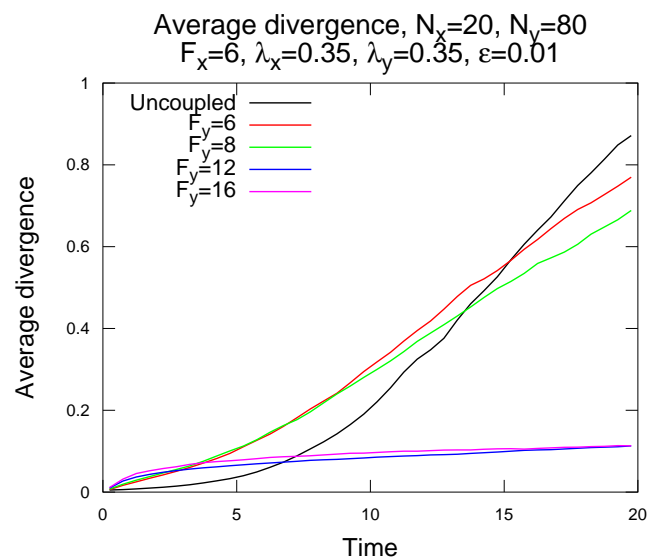

FIG. 6.2. Average divergence between perturbed and unperturbed running averages of the slow variables for the rescaled Lorenz model in (2.8) with the following parameters: $N_{x}=20, N_{y}=80$, $F_{x}=6, F_{y}=6,8,12$ and $16, \lambda_{x}=\lambda_{y}=0.35, \varepsilon=0.01$, as well as for the uncoupled rescaled Lorenz model with $N=20$ and $F=6$.
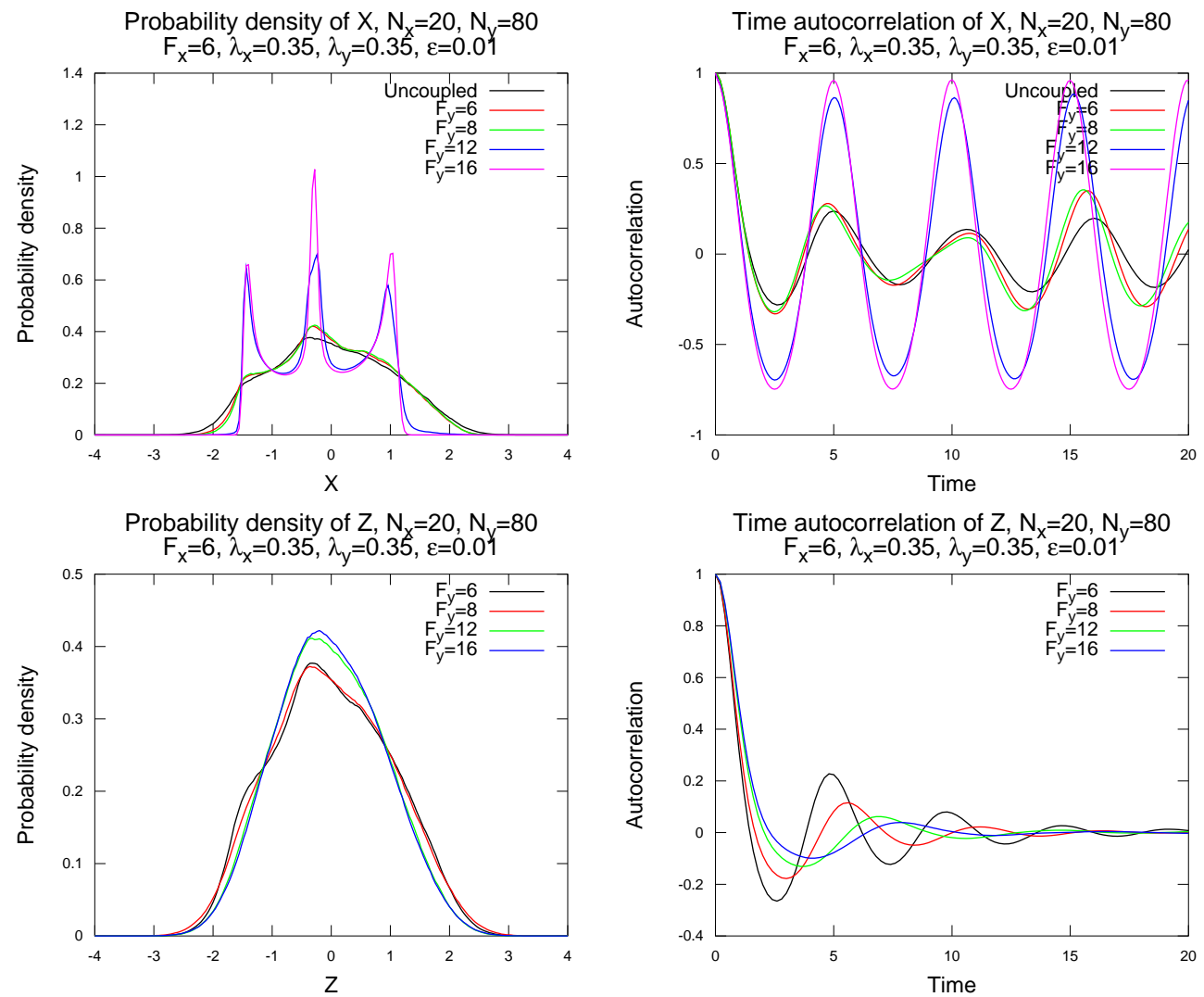

Fig. 6.3. Probability density functions and time autocorrelation functions for $\boldsymbol{x}$ of (2.8) and $\boldsymbol{z}$ of (2.9) (with free parameter $\boldsymbol{x}$ set to its mean value) with the following parameters: $N_{x}=20$, $N_{y}=80, F_{x}=6, F_{y}=6,8,12$ and $16, \lambda_{x}=\lambda_{y}=0.35, \varepsilon=0.01$. 
parameters $N_{x}=20, N_{y}=80, F_{x}=6, \lambda_{x}=\lambda_{y}=0.35, \varepsilon=0.01$, and compare it with the uncoupled rescaled Lorenz model in (2.7) with $N=20$ and $F=6$. In Figure 6.2 we show the average divergence of a perturbed trajectory from an unperturbed one for this set of parameters. Observe that while the slow dynamics for $F_{y}=6,8$ and for uncoupled dynamics with $F=6$ are clearly chaotic, for $F_{y}=12$ and greater values the abrupt transition occurs, where the difference between the perturbed and unperturbed $\boldsymbol{x}$ time series does not grow much beyond $10 \%$. The statistics for the same set of parameters are shown in Figure 6.3 and Table 6.1. Here, clearly the correlations and probability densities for the slow variables differ significantly for the chaotic and stable regimes, with the statistics for the uncoupled Lorenz model with $F=6$ being almost identical to those for the chaotic regimes with $F_{y}=6,8$. However, the chaos at slow variables is suppressed purely by the dynamical mechanism uncovered in this work; indeed, the mean state and variance of both the slow and fast variables do not change substantially enough to suppress chaos by creating a counteracting mean forcing term at the slow dynamics to suppress $F_{x}$ (see Table 6.1 for confirmation), while the time autocorrelation functions for the fast variables in Figure 6.3 with $\boldsymbol{x}$ set to the mean state decay faster for larger values of $F_{y}$, indicating stronger mixing. The key observation here is that the behavior of the uncoupled model with only slow variables in the same regime is deceiving - it is clearly chaotic, while the full two-scale model loses chaos at the slow variables in more turbulent regimes of the fast dynamics.

\section{Generalization onto the two-scale setting with stochastic noise and explicit time dependence}

For the simplicity of the presentation, the theory above was developed for a simplified setting in (2.1), which matched the structure of the two-scale rescaled Lorenz model. However, the real-world chaotic multiscale models often include the stochastic noise terms. Additionally, the explicit dependence on the range of different time scales is quite common in practice. As an example, one can look at the annual and diurnal time scales of the external solar forcing at a given physical location on the surface of the Earth. These time scales differ by two orders of magnitude. Below we develop the framework for a more general setting, which includes both the stochastic noise and explicit dependence on time.

Here, we modify the dynamical system in (2.1) by including the explicit time dependence on both the slow time scale $t$ and the fast time scale $t / \varepsilon$. Additionally, we include the noise terms at both the slow and fast variables. The dynamical system in (2.1) becomes a system of the Itô stochastic differential equations of the form

$$
\begin{gathered}
\mathrm{d} \boldsymbol{x}=\boldsymbol{F}(\boldsymbol{x}, \boldsymbol{y}, t, t / \varepsilon) \mathrm{d} t+\boldsymbol{\kappa}(\boldsymbol{x}, t, t / \varepsilon) \mathrm{d} \boldsymbol{V}_{t}, \\
\mathrm{~d} \boldsymbol{y}=\frac{1}{\varepsilon} \boldsymbol{G}(\boldsymbol{x}, \boldsymbol{y}, t, t / \varepsilon) \mathrm{d} t+\frac{1}{\sqrt{\varepsilon}} \boldsymbol{\sigma}(\boldsymbol{y}, t, t / \varepsilon) \mathrm{d} \boldsymbol{W}_{t} .
\end{gathered}
$$

Above, the terms $\boldsymbol{V}_{t}$ and $\boldsymbol{W}_{t}$ are $K_{x^{-}}$and $K_{y^{-}}$dimensional Wiener processes, respectively, for some positive integers $K_{x}$ and $K_{y}$, while $\boldsymbol{\kappa}(\boldsymbol{x}, t, t / \varepsilon)$ and $\boldsymbol{\sigma}(\boldsymbol{y}, t, t / \varepsilon)$ are $N_{x} \times K_{x}$ and $N_{y} \times K_{y}$ matrix-valued functions, respectively. Observe that here $\boldsymbol{\kappa}$ does not depend on $\boldsymbol{y}$, while $\boldsymbol{\sigma}$ does not depend on $\boldsymbol{x}$, as the coupling between the slow and fast variables must be deterministic to allow for total energy conservation.

In Section 3 we assumed that both the slow and fast dynamics in (2.1) do not depend explicitly on time, and that assumption rendered the fast limiting dynamics in (2.3) autonomous with the invariant distribution measure $\mu_{\boldsymbol{x}}$. Since the dynamics 
in (8.1) explicitly depend on $t / \varepsilon$, the fast limiting dynamics as $\varepsilon \rightarrow \infty$ (the analogue of that in (2.3)) is non-autonomous:

$$
\mathrm{d} \boldsymbol{z}=\boldsymbol{G}(\boldsymbol{x}, \boldsymbol{z}, t, s) \mathrm{d} s+\boldsymbol{\sigma}(\boldsymbol{z}, t, s) \mathrm{d} \boldsymbol{W}_{s} .
$$

Here, $\boldsymbol{x}$ and $t$ are the constant parameters, and the invariant measure $\mu$ for the dynamics of $\boldsymbol{z}(s)$ above may no longer exist because of the explicit dependence of (8.2) on $s$. However, practice shows that, from the physical perspective, averages exist even in a non-autonomous system (where the averaging is assumed to be with respect to the time $s$ ). Usually, the reason for that is that the way $\boldsymbol{F}, \boldsymbol{G}, \boldsymbol{\kappa}$, and $\boldsymbol{\sigma}$ depend on $s$ is bounded, so in the limit as $s \rightarrow \infty$, these functions "average out" over some bounded subset of the phase space instead of blowing up to infinity (as a simplest example, one could think of a periodic dependence on $s$ ). In Appendix $\mathrm{C}$ we set up a suitable mathematical framework, in which the above arguments are arranged in a more formal fashion through a combination of the appropriate skew product flows (see, for example, $[9,10]$ and references therein). The resulting averaged system is given by

$$
\begin{gathered}
\mathrm{d} \boldsymbol{x}=\langle\boldsymbol{F}\rangle(\boldsymbol{x}, t) \mathrm{d} t+\langle\boldsymbol{\kappa}\rangle(\boldsymbol{x}, t) \mathrm{d} \boldsymbol{V}_{t}, \\
\langle\boldsymbol{F}\rangle(\boldsymbol{x}, t)=\lim _{r \rightarrow \infty} \frac{1}{r} \int_{0}^{r} \boldsymbol{F}(\boldsymbol{x}, \boldsymbol{z}(s), t, s) \mathrm{d} s \\
\langle\boldsymbol{\kappa}\rangle(\boldsymbol{x}, t)\langle\boldsymbol{\kappa}\rangle(\boldsymbol{x}, t)^{T}=\lim _{r \rightarrow \infty} \frac{1}{r} \int_{0}^{r} \boldsymbol{\kappa}(\boldsymbol{x}, t, s) \boldsymbol{\kappa}(\boldsymbol{x}, t, s)^{T} \mathrm{~d} s . \\
\mathrm{d} \boldsymbol{T} \boldsymbol{X}_{\boldsymbol{x}}^{t_{0}, t}=\left[\left(\left\langle\frac{\partial \boldsymbol{F}}{\partial \boldsymbol{x}}\right\rangle(\boldsymbol{x}, t)+\boldsymbol{H}(\boldsymbol{x}, t)\right) \mathrm{d} t+\frac{\partial\langle\boldsymbol{\kappa}\rangle(\boldsymbol{x}, t)}{\partial \boldsymbol{x}} \mathrm{d} \boldsymbol{V}_{t}\right] \boldsymbol{T} \boldsymbol{X}_{\boldsymbol{x}}^{t_{0}, t}, \\
\left\langle\frac{\partial \boldsymbol{F}}{\partial \boldsymbol{x}}\right\rangle(\boldsymbol{x}, t)=\lim _{r \rightarrow \infty} \frac{1}{r} \int_{0}^{r} \frac{\partial \boldsymbol{F}}{\partial \boldsymbol{x}}(\boldsymbol{x}, \boldsymbol{z}(s), t, s) \mathrm{d} s, \\
\boldsymbol{H}(\boldsymbol{x}, t)=\int_{0}^{\infty}\left(\lim _{r \rightarrow \infty} \frac{1}{r} \int_{0}^{r} \frac{\partial \boldsymbol{F}}{\partial \boldsymbol{y}}(\boldsymbol{x}, \boldsymbol{z}(\tau+s), t, \tau+s) \times\right. \\
\left.\times \boldsymbol{T}_{\boldsymbol{x}, t, \boldsymbol{z}(\tau)}^{\tau, s} \frac{\partial \boldsymbol{G}}{\partial \boldsymbol{x}}(\boldsymbol{x}, \boldsymbol{z}(\tau), t, \tau) \mathrm{d} \tau\right) \mathrm{d} s,
\end{gathered}
$$

where $\boldsymbol{T}_{\boldsymbol{x}, t, \boldsymbol{z}(\tau)}^{\tau, s}$ is the tangent map of (8.2) at $\boldsymbol{z}(\tau)$ to $\tau+s$.

Now, following Section 4, we write (8.1) in the linear energy-preserving coupling form:

$\mathrm{d} \boldsymbol{x}=\left[\boldsymbol{f}(\boldsymbol{x}, t, t / \varepsilon)+\boldsymbol{S}_{x}^{-1 / 2} \boldsymbol{L}(t) \boldsymbol{S}_{y}^{1 / 2} \boldsymbol{y}\right] \mathrm{d} t+\boldsymbol{\kappa}(\boldsymbol{x}, t, t / \varepsilon) \mathrm{d} \boldsymbol{V}_{t}$,

$\mathrm{d} \boldsymbol{y}=\frac{1}{\varepsilon}\left[\boldsymbol{g}(\boldsymbol{y}, t, t / \varepsilon)-\boldsymbol{S}_{y}^{-1 / 2} \boldsymbol{L}^{T}(t) \boldsymbol{S}_{x}^{1 / 2} \boldsymbol{x}\right] \mathrm{d} t+\frac{1}{\sqrt{\varepsilon}} \boldsymbol{\sigma}(\boldsymbol{y}, t, t / \varepsilon) \mathrm{d} \boldsymbol{W}_{t}$.

Here, observe that the linear coupling matrix $\boldsymbol{L}$ may depend on the slow time $t$, $\boldsymbol{L}=\boldsymbol{L}(t)$. With (8.4) it is not hard to see that that averaged dynamics for the slow tangent map are given by

$$
\begin{array}{r}
\mathrm{d} \boldsymbol{T} \boldsymbol{X}_{\boldsymbol{x}}^{t_{0}, t}=\left[\left(\left\langle\frac{\partial \boldsymbol{f}}{\partial \boldsymbol{x}}\right\rangle(\boldsymbol{x}, t)-\boldsymbol{S}_{x}^{-1 / 2} \boldsymbol{L}(t) \boldsymbol{S}_{y}^{1 / 2} \mathcal{C}(\boldsymbol{x}, t) \boldsymbol{S}_{y}^{-1 / 2} \boldsymbol{L}^{T}(t) \boldsymbol{S}_{x}^{1 / 2}\right) \mathrm{d} t\right. \\
\left.+\frac{\partial\langle\boldsymbol{\kappa}\rangle(\boldsymbol{x}, t)}{\partial \boldsymbol{x}} \mathrm{d} \boldsymbol{V}_{t}\right] \boldsymbol{T} \boldsymbol{X}_{\boldsymbol{x}}^{t_{0}, t}
\end{array}
$$




$$
\begin{gathered}
\left\langle\frac{\partial \boldsymbol{f}}{\partial \boldsymbol{x}}\right\rangle(\boldsymbol{x}, t)=\lim _{r \rightarrow \infty} \frac{1}{r} \int_{0}^{r} \frac{\partial \boldsymbol{f}}{\partial \boldsymbol{x}}(\boldsymbol{x}, t, s) \mathrm{d} s \\
\mathcal{C}(\boldsymbol{x}, t)=\int_{0}^{\infty}\left(\lim _{r \rightarrow \infty} \frac{1}{r} \int_{0}^{r} \boldsymbol{T}_{\boldsymbol{x}, t, \boldsymbol{z}(\tau)}^{\tau, s} \mathrm{~d} \tau\right) \mathrm{d} s .
\end{gathered}
$$

Here we can see that the situation is completely analogous to what was found in Sections $4-5$, that is, the positive-definiteness of $\mathcal{C}(\boldsymbol{x}, t)$ in the canonical energy coordinates has a damping effect on the slow averaged tangent dynamics in (8.5), thereby reducing chaos at the slow variables.

9. Nonlinear energy-preserving coupling with dependence on the slow and fast variables

Recall that in Sections 4 and 8 we assumed that the matrix $\boldsymbol{L}$ in the energy preserving coupling is either a constant matrix (Section 4), or is a function of only the slow time $t$ (Section 8). However, for the purpose of the energy preservation, $\boldsymbol{L}$ can be an arbitrary $N_{x} \times N_{y}$ matrix function $\boldsymbol{L}(\boldsymbol{x}, \boldsymbol{y}, t, t / \varepsilon)$. Below we briefly outline the situation when $\boldsymbol{L}$ is a function of $\boldsymbol{x}, \boldsymbol{y}, t$, and $t / \varepsilon$.

In such a case, we can extract the slow part $\boldsymbol{L}(t)$ of the energy-preserving coupling $\boldsymbol{L}(\boldsymbol{x}, \boldsymbol{y}, t, t / \varepsilon)$ by time-averaging over the fast variables on the trajectory $\boldsymbol{x}(t)$ :

$$
\begin{gathered}
\boldsymbol{L}(t)=\lim _{r \rightarrow \infty} \frac{1}{r} \int_{0}^{r} \boldsymbol{L}(\boldsymbol{x}(t), \boldsymbol{z}(s), t, s) \mathrm{d} s, \\
\boldsymbol{L}(\boldsymbol{x}, \boldsymbol{y}, t, s)=\boldsymbol{L}(t)+\boldsymbol{L}^{\prime}(\boldsymbol{x}, \boldsymbol{y}, t, s),
\end{gathered}
$$

where $\boldsymbol{x}(t)$ is the solution of (8.4), and $\boldsymbol{z}(s)$ is the solution of (8.2) for given $\boldsymbol{x}$ and $t$. Thus, the term $\boldsymbol{L}^{\prime}(\boldsymbol{x}, \boldsymbol{y}, t, s)$ in (9.1) represents rapid oscillations with zero mean state around the slowly varying $\boldsymbol{L}(t)$ :

$$
\lim _{r \rightarrow \infty} \frac{1}{r} \int_{0}^{r} \boldsymbol{L}^{\prime}(\boldsymbol{x}(t), \boldsymbol{z}(s), t, s) \mathrm{d} s=\mathbf{0} .
$$

With (9.1), the equations in (8.4) become

$$
\begin{gathered}
\mathrm{d} \boldsymbol{x}=\left[\boldsymbol{f}(\boldsymbol{x}, \boldsymbol{y}, t, t / \varepsilon)+\boldsymbol{S}_{x}^{-1 / 2} \boldsymbol{L}(t) \boldsymbol{S}_{y}^{1 / 2} \boldsymbol{y}\right] \mathrm{d} t+\boldsymbol{\kappa}(\boldsymbol{x}, t, t / \varepsilon) \mathrm{d} \boldsymbol{V}_{t}, \\
\mathrm{~d} \boldsymbol{y}=\frac{1}{\varepsilon}\left[\boldsymbol{g}(\boldsymbol{x}, \boldsymbol{y}, t, t / \varepsilon)-\boldsymbol{S}_{y}^{-1 / 2} \boldsymbol{L}^{T}(t) \boldsymbol{S}_{x}^{1 / 2} \boldsymbol{x}\right] \mathrm{d} t+\frac{1}{\sqrt{\varepsilon}} \boldsymbol{\sigma}(\boldsymbol{y}, t, t / \varepsilon) \mathrm{d} \boldsymbol{W}_{t},
\end{gathered}
$$

where we denote

$$
\begin{gathered}
\boldsymbol{f}(\boldsymbol{x}, \boldsymbol{y}, t, s)=\boldsymbol{f}(\boldsymbol{x}, t, s)+\boldsymbol{S}_{x}^{-1 / 2} \boldsymbol{L}^{\prime}(\boldsymbol{x}, \boldsymbol{y}, t, s) \boldsymbol{S}_{y}^{1 / 2} \boldsymbol{y} \\
\boldsymbol{g}(\boldsymbol{x}, \boldsymbol{y}, t, s)=\boldsymbol{g}(\boldsymbol{y}, t, s)-\boldsymbol{S}_{y}^{-1 / 2} \boldsymbol{L}^{\prime T}(\boldsymbol{x}, \boldsymbol{y}, t, s) \boldsymbol{S}_{x}^{1 / 2} \boldsymbol{x}
\end{gathered}
$$

With the above changes, the right-hand side of (C.6) acquires a new term:

$$
\begin{array}{r}
\mathrm{d} \boldsymbol{T} \boldsymbol{X}_{\boldsymbol{x}}^{t_{0}, t}=\left[\left(\left\langle\frac{\partial \boldsymbol{f}}{\partial \boldsymbol{x}}\right\rangle(\boldsymbol{x}, t)-\boldsymbol{S}_{x}^{-1 / 2} \boldsymbol{L}(t) \boldsymbol{S}_{y}^{1 / 2} \mathcal{C}(\boldsymbol{x}, t) \boldsymbol{S}_{y}^{-1 / 2} \boldsymbol{L}^{T}(t) \boldsymbol{S}_{x}^{1 / 2}\right.\right. \\
\left.\left.+\int_{0}^{\infty} \boldsymbol{H}^{\prime}(\boldsymbol{x}, t, s)\right) \mathrm{d} s+\frac{\partial\langle\boldsymbol{\kappa}\rangle(\boldsymbol{x}, t)}{\partial \boldsymbol{x}} \mathrm{d} \boldsymbol{V}_{t}\right] \boldsymbol{T} \boldsymbol{X}_{\boldsymbol{x}}^{t_{0}, t}
\end{array}
$$




$$
\begin{aligned}
& \boldsymbol{H}^{\prime}(\boldsymbol{x}, t, s)=\boldsymbol{S}_{x}^{-1 / 2} \boldsymbol{L}(t) \boldsymbol{S}_{y}^{1 / 2}\left[\lim _{r \rightarrow \infty} \frac{1}{r} \int_{0}^{r} \boldsymbol{T}_{\boldsymbol{x}, t, \boldsymbol{z}(\tau)}^{\tau, s} \frac{\partial \boldsymbol{g}}{\partial \boldsymbol{x}}(\boldsymbol{x}, \boldsymbol{z}(\tau), t, \tau) \mathrm{d} \tau\right] \\
&- {\left[\lim _{r \rightarrow \infty} \frac{1}{r} \int_{0}^{r} \frac{\partial \boldsymbol{f}}{\partial \boldsymbol{z}}(\boldsymbol{x}, \boldsymbol{z}(\tau+s), t, \tau+s) \boldsymbol{T}_{\boldsymbol{x}, t, \boldsymbol{z}(\tau)}^{\tau, s} \mathrm{~d} \tau\right] \boldsymbol{S}_{y}^{-1 / 2} \boldsymbol{L}^{T}(t) \boldsymbol{S}_{x}^{1 / 2} } \\
&+ \lim _{r \rightarrow \infty} \frac{1}{r} \int_{0}^{r} \frac{\partial \boldsymbol{f}}{\partial \boldsymbol{z}}(\boldsymbol{x}, \boldsymbol{z}(\tau+s), t, \tau+s) \boldsymbol{T}_{\boldsymbol{x}, t, \boldsymbol{z}(\tau)}^{\tau, s} \frac{\partial \boldsymbol{g}}{\partial \boldsymbol{x}}(\boldsymbol{x}, \boldsymbol{z}(\tau), t, \tau) \mathrm{d} \tau
\end{aligned}
$$

It is not known at present how the new term $\boldsymbol{H}^{\prime}(\boldsymbol{x}, t, s)$ is going to affect the dynamics, however, the chaos-suppressing term $\mathcal{C}(\boldsymbol{x}, t)$ is nonetheless retained by the dynamics. One can presume that if the effect of the new term is small, the chaos suppression through $\mathcal{C}(\boldsymbol{x}, t)$ would still be observed. At this point, it is hardly possible to say anything about the effect of $\boldsymbol{H}^{\prime}(\boldsymbol{x}, t, s)$ without making additional assumptions about its structure. Clearly, the answer to this question depends on a particular type of energy-preserving coupling used in the model. While a general coupling of the form in (9.1) might not necessarily promote the suppression of chaos at the slow variables, there could be additional restrictions on $\boldsymbol{L}^{\prime}(\boldsymbol{x}, \boldsymbol{y}, t, s)$ imposed by the physics of a particular model under consideration. In future work, the author intends to derive suitable classes of a more general nonlinear energy-preserving coupling which nonetheless promotes the suppression of chaos at the slow variables.

\section{Invariance with respect to time-scaling and independence of $\varepsilon$}

Throughout the work, it was presumed that the dependence of the model on the time-scale separation parameter $\varepsilon$ is explicit. However, often in real-world situations the parameter $\varepsilon$ is not present explicitly, but it is known from the observations that there is a subset of the "slow" variables in the system, while the rest of the variables are "fast". It turns out that the framework, developed here, also does not explicitly depend on $\varepsilon$. Observe that the averages in (C.6) are invariant under the time-rescaling of the limiting dynamics in (8.2) as

$$
\mathrm{d} \boldsymbol{z}=\alpha \boldsymbol{G}(\boldsymbol{x}, \boldsymbol{z}, t, s) \mathrm{d} s+\sqrt{\alpha} \boldsymbol{\sigma}(\boldsymbol{z}, t, s) \mathrm{d} \boldsymbol{W}_{s},
$$

where $\alpha>0$ is an arbitrary parameter. Setting $\alpha=\varepsilon^{-1}$ yields

$$
\mathrm{d} \boldsymbol{z}=\frac{1}{\varepsilon} \boldsymbol{G}(\boldsymbol{x}, \boldsymbol{z}, t, s) \mathrm{d} s+\frac{1}{\sqrt{\varepsilon}} \boldsymbol{\sigma}(\boldsymbol{z}, t, s) \mathrm{d} \boldsymbol{W}_{s},
$$

which is obtained directly from the fast equation in (8.1b) by setting $\boldsymbol{x}$ and $t$ as constant parameters. Due to this property, the explicit knowledge of the time-scale separation parameter $\varepsilon$ is not necessary; for all practical purposes, one can assume that the original slow-fast system in (8.1) is supplied, without the time-scale separation parameter $\varepsilon$, in the form

$$
\begin{gathered}
\mathrm{d} \boldsymbol{x}=\boldsymbol{F}(\boldsymbol{x}, \boldsymbol{y}, t, t) \mathrm{d} t+\boldsymbol{\kappa}(\boldsymbol{x}, t, t) \mathrm{d} \boldsymbol{V}_{t}, \\
\mathrm{~d} \boldsymbol{y}=\boldsymbol{G}(\boldsymbol{x}, \boldsymbol{y}, t, t) \mathrm{d} t+\boldsymbol{\sigma}(\boldsymbol{y}, t, t) \mathrm{d} \boldsymbol{W}_{t},
\end{gathered}
$$

for which it is known that $\boldsymbol{x}$ is the set of slow variables and $\boldsymbol{y}$ is the set of fast variables, and that the dependence on the first $t$-parameter in each function is slow, while the dependence on the second $t$-parameter is fast. The limiting dynamics in (8.2) are thus obtained by setting $\boldsymbol{x}$ and first $t$-parameter in (10.3b) to constants, and the averaged slow tangent dynamics in (C.6) remain in effect with no corrections. 


\section{Summary}

In this work we study the effect of the fast rapidly mixing dynamics on the chaos at slow variables in a two-scale system with a deterministic linear energy-preserving coupling. A suitable theory is developed by applying the averaging formalism to the tangent dynamics of the system, and it is found that the linear energy-preserving coupling creates a systematic damping effect on the chaos at the slow scales when the fast dynamics is rapidly mixing (the precise criterion is the positive-definiteness of the infinite-time linear response to a small constant external forcing at the fast scales), which can also be interpreted as the linear stability property of the long-term mean state of the fast limiting system under the external perturbations. It is suggested that this property must strongly depend on the ratio of the autocorrelation time and the advection time scale at the fast variables, however, a systematic study of this proposition is left for the future work. The effect of the chaos suppression at the slow variables through the rapidly mixing fast dynamics is systematically demonstrated for the two-scale Lorenz model, which is also appropriately rescaled so that the adjustments for the mixing regime at the fast variables do not affect the mean state and variance of both the slow and fast variables. In particular, it is shown through the numerical experiment that the uncoupled slow dynamics, where the fast variables are replaced with their averages (which are zeros in the rescaled Lorenz model), may remain chaotic, while the full coupled system loses chaos and becomes completely predictable at the slow scales as the dynamics at the fast scales become more turbulent. The theory is also systematically generalized onto the more general setting with nonautonomous dynamics and stochastic noise at both the slow and fast variables.

Future work. Clearly, the present work, while uncovering a dynamical mechanism for the chaos suppression at the slow variables in a multiscale system, does not yet provide any systematic quantification of the chaos suppression effect, which will have to be addressed in the future work. Below we sketch a few key directions, immediately emerging from the results of the present study.

- Is there a simple direct quantitative connection between the chaos at the slow variables and the infinite-time linear response at the fast variables? Above, while the dynamical mechanism of the chaos suppression is discussed, no quantitative connections between the Lyapunov characteristic exponents of the slow averaged dynamics and the eigenvalues of the symmetric part of the infinite-time linear response at the fast variables are investigated. Are there any simple direct estimates of the chaos reduction at the slow variables through the autocorrelation times of the fast variables?

- Is it possible to estimate the chaos suppression effect from the autocorrelation time/advection time scale ratio? Above we briefly formulated the hypothesis that the suppression of chaos at the slow variables could be amplified by a large ratio of the advection time scale to the autocorrelation time at the fast variables. If this hypothesis is correct, would it be possible to make an estimate of the chaos suppression effect by knowing the ratio of the advection time scale to the autocorrelation time, perhaps through the theory of turbulence?

- What if the energy-preserving coupling is nonlinear? We briefly sketched that if the energy-preserving coupling is nonlinear, then an additional term appears in the dynamics of the slow averaged tangent map, and it is not immediately clear whether it would create a damping or amplify- 
ing slow chaos effect. Can one identify suitable physically relevant classes of nonlinear coupling which suppresses chaos at the slow variables?

- What happens in more sophisticated models? Are the fully coupled models less chaotic at the slow variables than the uncoupled low-frequency variability models? If so, could the global climate be generally less chaotic than we presently tend to think? Could the predictive skill be improved by appropriately adjusting the uncoupled models to match the slow chaos properties of the coupled dynamics?

The author intends to address these problems in the future work, possibly in collaboration with atmospheric scientists.

Acknowledgment. The author thanks Ibrahim Fatkullin for fruitful discussions. The author is supported by the National Science Foundation CAREER grant DMS-0845760, and the Office of Naval Research grants N00014-09-0083 and 25-74200F6607.

Appendix A. Infinite time linear response to changes in $x$.

Let $\mu_{\boldsymbol{x}}$ be an invariant measure for the dynamics given by

$$
\frac{\mathrm{d} \boldsymbol{z}}{\mathrm{d} t}=\boldsymbol{G}(\boldsymbol{x}, \boldsymbol{z})
$$

with flow $\phi_{\boldsymbol{x}}^{t}$ and ergodic attractor $\mathcal{A}_{\boldsymbol{x}}$, where $\boldsymbol{x}$ is a fixed parameter. Let $B(\boldsymbol{z})$ be a differentiable function of $\boldsymbol{z}$, and let $\langle B\rangle_{\boldsymbol{x}}$ denote the average

$$
\langle B\rangle_{\boldsymbol{x}}=\int_{\mathcal{A}_{\boldsymbol{x}}} B(\boldsymbol{z}) \mathrm{d} \mu_{\boldsymbol{x}}(\boldsymbol{z})
$$

Our goal here is to compute the derivative of $\langle B\rangle_{\boldsymbol{x}}$ with respect to $\boldsymbol{x}$ (if it exists):

$$
\frac{\mathrm{d}\langle B\rangle_{\boldsymbol{x}}}{\mathrm{d} \boldsymbol{x}}=\int_{\mathcal{A}_{\boldsymbol{x}}} B(\boldsymbol{z}) \mathrm{d} \mu_{\boldsymbol{x}}^{\prime}(\boldsymbol{z}) .
$$

Here we are going to use the linear response theory from $[1-3,5-7,20,25,28]$. Let us assume that, at the initial time $t=0$, there is a statistical ensemble of points $\boldsymbol{z}$, distributed according to $\mu_{\boldsymbol{x}}$, and, at the same time, there is a change $\boldsymbol{x}+\delta \boldsymbol{x}$ in (A.1). Then, one can treat the difference between the averages along the perturbed and unperturbed trajectories of (A.1), emerging from the initial statistical ensemble, as time goes to infinity, as the derivative in (A.3):

$$
\begin{aligned}
& \int_{\mathcal{A}_{\boldsymbol{x}}} B(\boldsymbol{z}) \mathrm{d} \mu_{\boldsymbol{x}}^{\prime}(\boldsymbol{z}) \delta \boldsymbol{x}+O\left(\|\delta \boldsymbol{x}\|^{2}\right) \\
= & \lim _{t \rightarrow \infty} \int_{\mathcal{A}_{\boldsymbol{x}}}\left[B\left(\phi_{\boldsymbol{x}+\delta \boldsymbol{x}}^{t} \boldsymbol{z}\right)-B\left(\phi_{\boldsymbol{x}}^{t} \boldsymbol{z}\right)\right] \mathrm{d} \mu_{\boldsymbol{x}}(\boldsymbol{z}) .
\end{aligned}
$$

For the above relation to hold, the structural stability of $\mu_{\boldsymbol{x}}$ under the changes in $\boldsymbol{x}$ is required. It is known that uniformly hyperbolic diffeomorphisms on the whole phase space of (A.1) (Anosov) or just its nonwandering set (Axiom A) are structurally stable $[13,26-28,33]$. The integral in the right-hand side above is given by

$$
\begin{aligned}
& \int_{\mathcal{A}_{\boldsymbol{x}}}\left[B\left(\phi_{\boldsymbol{x}+\delta \boldsymbol{x}}^{t} \boldsymbol{z}\right)-B\left(\phi_{\boldsymbol{x}}^{t} \boldsymbol{z}\right)\right] \mathrm{d} \mu_{\boldsymbol{x}}(\boldsymbol{z}) \\
= & \int_{\mathcal{A}_{\boldsymbol{x}}}\left[\nabla B\left(\phi_{\boldsymbol{x}}^{t} \boldsymbol{z}\right) \frac{\partial}{\partial \boldsymbol{x}} \phi_{\boldsymbol{x}}^{t} \boldsymbol{z}\right] \mathrm{d} \mu_{\boldsymbol{x}}(\boldsymbol{z}) \delta \boldsymbol{x}+O\left(\|\delta \boldsymbol{x}\|^{2}\right) .
\end{aligned}
$$


To compute the derivative of the flow $\phi_{\boldsymbol{x}}^{t} \boldsymbol{z}$ in $\boldsymbol{x}$, we use the fact that it is a solution of (A.1), and therefore satisfies

$$
\frac{\partial}{\partial t} \phi_{\boldsymbol{x}}^{t} \boldsymbol{z}=\boldsymbol{G}\left(\boldsymbol{x}, \phi_{\boldsymbol{x}}^{t} \boldsymbol{z}\right), \quad \frac{\partial}{\partial t} \phi_{\boldsymbol{x}+\delta \boldsymbol{x}}^{t} \boldsymbol{z}=\boldsymbol{G}\left(\boldsymbol{x}+\delta \boldsymbol{x}, \phi_{\boldsymbol{x}+\delta \boldsymbol{x}}^{t} \boldsymbol{z}\right) .
$$

Subtracting one identity from another and Taylor-expanding in $\delta \boldsymbol{x}$, we obtain

$$
\frac{\partial}{\partial t}\left(\frac{\partial}{\partial \boldsymbol{x}} \phi_{\boldsymbol{x}}^{t} \boldsymbol{z}\right)=\frac{\partial \boldsymbol{G}}{\partial \boldsymbol{z}}\left(\boldsymbol{x}, \phi_{\boldsymbol{x}}^{t} \boldsymbol{z}\right)\left(\frac{\partial}{\partial \boldsymbol{x}} \phi_{\boldsymbol{x}}^{t} \boldsymbol{z}\right)+\frac{\partial \boldsymbol{G}}{\partial \boldsymbol{x}}\left(\boldsymbol{x}, \phi_{\boldsymbol{x}}^{t} \boldsymbol{z}\right)+O(\|\delta \boldsymbol{x}\|) .
$$

By sending $\delta \boldsymbol{x}$ to zero, the formal solution to the above equation with zero initial condition is given by Duhamel's principle:

$$
\frac{\partial}{\partial \boldsymbol{x}} \phi_{\boldsymbol{x}}^{t} \boldsymbol{z}=\int_{0}^{t} \boldsymbol{T}_{\boldsymbol{x}, \boldsymbol{z}}^{t-s} \frac{\partial \boldsymbol{G}}{\partial \boldsymbol{x}}\left(\boldsymbol{x}, \phi_{\boldsymbol{x}}^{s} \boldsymbol{z}\right) \mathrm{d} s
$$

where $\boldsymbol{T}_{\boldsymbol{x}, \boldsymbol{z}}^{t}$ is the tangent map of $\phi_{\boldsymbol{x}}^{t} \boldsymbol{z}$

$$
\boldsymbol{T}_{\boldsymbol{x}, \boldsymbol{z}}^{t}=\frac{\partial}{\partial \boldsymbol{z}} \phi_{\boldsymbol{x}}^{t} \boldsymbol{z}
$$

satisfying

$$
\frac{\partial}{\partial t} \boldsymbol{T}_{\boldsymbol{x}, \boldsymbol{z}}^{t}=\frac{\partial \boldsymbol{G}}{\partial \boldsymbol{z}}\left(\boldsymbol{x}, \phi_{\boldsymbol{x}}^{\tau} \boldsymbol{z}\right) \boldsymbol{T}_{\boldsymbol{x}, \boldsymbol{z}}^{t}
$$

Substituting the above equation into (A.5), we obtain

$$
\begin{aligned}
& \int_{\mathcal{A}_{\boldsymbol{x}}}\left[B\left(\phi_{\boldsymbol{x}+\delta \boldsymbol{x}}^{t} \boldsymbol{z}\right)-B\left(\phi_{\boldsymbol{x}}^{t} \boldsymbol{z}\right)\right] \mathrm{d} \mu_{\boldsymbol{x}}(\boldsymbol{z}) \\
= & \int_{0}^{t} \int_{\mathcal{A}_{\boldsymbol{x}}}\left[\nabla B\left(\phi_{\boldsymbol{x}}^{t} \boldsymbol{z}\right) \boldsymbol{T}_{\boldsymbol{x}, \boldsymbol{z}}^{t-s} \frac{\partial \boldsymbol{G}}{\partial \boldsymbol{x}}\left(\boldsymbol{x}, \phi_{\boldsymbol{x}}^{s} \boldsymbol{z}\right)\right] \mathrm{d} \mu_{\boldsymbol{x}}(\boldsymbol{z}) \mathrm{d} s \delta \boldsymbol{x}+O\left(\|\delta \boldsymbol{x}\|^{2}\right) .
\end{aligned}
$$

By using the fact that $\mu_{\boldsymbol{x}}$ is the invariant measure for $\phi_{\boldsymbol{x}}^{t}$, we obtain

$$
\begin{aligned}
& \int_{\mathcal{A}_{\boldsymbol{x}}}\left[B\left(\phi_{\boldsymbol{x}+\delta \boldsymbol{x}}^{t} \boldsymbol{z}\right)-B\left(\phi_{\boldsymbol{x}}^{t} \boldsymbol{z}\right)\right] \mathrm{d} \mu_{\boldsymbol{x}}(\boldsymbol{z}) \\
= & \int_{0}^{t} \int_{\mathcal{A}_{\boldsymbol{x}}}\left[\nabla B\left(\phi_{\boldsymbol{x}}^{t-s} \boldsymbol{z}\right) \boldsymbol{T}_{\boldsymbol{x}, \boldsymbol{z}}^{t-s} \frac{\partial \boldsymbol{G}}{\partial \boldsymbol{x}}(\boldsymbol{x}, \boldsymbol{z})\right] \mathrm{d} \mu_{\boldsymbol{x}}(\boldsymbol{z}) \mathrm{d} s \delta \boldsymbol{x}+O\left(\|\delta \boldsymbol{x}\|^{2}\right) \\
= & \int_{0}^{t} \int_{\mathcal{A}_{\boldsymbol{x}}}\left[\nabla B\left(\phi_{\boldsymbol{x}}^{s} \boldsymbol{z}\right) \boldsymbol{T}_{\boldsymbol{x}, \boldsymbol{z}}^{s} \frac{\partial \boldsymbol{G}}{\partial \boldsymbol{x}}(\boldsymbol{x}, \boldsymbol{z})\right] \mathrm{d} \mu_{\boldsymbol{x}}(\boldsymbol{z}) \mathrm{d} s \delta \boldsymbol{x}+O\left(\|\delta \boldsymbol{x}\|^{2}\right) .
\end{aligned}
$$

Comparing the above relation with (A.4), we obtain, as $\delta \boldsymbol{x} \rightarrow 0$,

$$
\int_{\mathcal{A}_{\boldsymbol{x}}} B(\boldsymbol{z}) \mathrm{d} \mu_{\boldsymbol{x}}^{\prime}(\boldsymbol{z})=\int_{0}^{\infty} \int_{\mathcal{A}_{\boldsymbol{x}}}\left[\nabla B\left(\phi_{\boldsymbol{x}}^{s} \boldsymbol{z}\right) \boldsymbol{T}_{\boldsymbol{x}, \boldsymbol{z}}^{s} \frac{\partial \boldsymbol{G}}{\partial \boldsymbol{x}}(\boldsymbol{x}, \boldsymbol{z})\right] \mathrm{d} \mu_{\boldsymbol{x}}(\boldsymbol{z}) \mathrm{d} s .
$$

By using ergodicity of $\mu_{\boldsymbol{x}}$, we can replace the measure averages above with time averages over a long-term trajectory $\boldsymbol{z}(t)$ of (A.1):

$$
\int_{\mathcal{A}_{\boldsymbol{x}}} B(\boldsymbol{z}) \mathrm{d} \mu_{\boldsymbol{x}}^{\prime}(\boldsymbol{z})=\int_{0}^{\infty}\left(\lim _{r \rightarrow \infty} \frac{1}{r} \int_{0}^{r}\left[\nabla B(\boldsymbol{z}(\tau+s)) \boldsymbol{T}_{\boldsymbol{x}, \boldsymbol{z}(\tau)}^{s} \frac{\partial \boldsymbol{G}}{\partial \boldsymbol{x}}(\boldsymbol{x}, \boldsymbol{z}(\tau))\right] \mathrm{d} \tau\right) \mathrm{d} s
$$


In terms of the linear response theory $[1-3,5-7,20,25,28]$, the above expression is the infinite time linear response of $\langle B\rangle$ to the changes in $\boldsymbol{x}$.

Appendix B. Infinite time linear response of the mean state to constant additive perturbation.

Let $\mu$ be an invariant measure for the dynamics given by

$$
\frac{\mathrm{d} \boldsymbol{z}}{\mathrm{d} t}=\boldsymbol{G}(\boldsymbol{z})
$$

with flow $\phi^{t}$ and ergodic attractor $\mathcal{A}$. Let $\langle\boldsymbol{z}\rangle$ be the mean state of (B.1):

$$
\langle\boldsymbol{z}\rangle=\int_{\mathcal{A}} \boldsymbol{z} \mathrm{d} \mu(\boldsymbol{z}) .
$$

Now, assume that (B.1) is perturbed by a constant additive perturbation $\delta \boldsymbol{f}$,

$$
\frac{\mathrm{d} \boldsymbol{z}}{\mathrm{d} t}=\boldsymbol{G}(\boldsymbol{z})+\delta \boldsymbol{f}
$$

with perturbed flow given by $\phi^{* t}$. Our goal here is to compute the infinite time linear response of $\langle\boldsymbol{z}\rangle$. Following Appendix A, we write

$$
\frac{\partial}{\partial t} \phi^{t} \boldsymbol{z}=\boldsymbol{G}\left(\phi^{t} \boldsymbol{z}\right), \quad \frac{\partial}{\partial t} \phi^{* t} \boldsymbol{z}=\boldsymbol{G}\left(\phi^{* t} \boldsymbol{z}\right)+\delta \boldsymbol{f} .
$$

Let the difference between the perturbed and unperturbed flows be given as $\delta \phi^{t} \boldsymbol{z}$. Now, taking the difference between the two relations above, we obtain

$$
\begin{aligned}
\frac{\partial}{\partial t}\left(\delta \phi^{t} \boldsymbol{z}\right) & =\boldsymbol{G}\left(\phi^{t} \boldsymbol{z}+\delta \phi^{t} \boldsymbol{z}\right)-\boldsymbol{G}\left(\phi^{t} \boldsymbol{z}\right)+\delta \boldsymbol{f} \\
& =\nabla \boldsymbol{G}\left(\phi^{t} \boldsymbol{z}\right) \delta \phi^{t} \boldsymbol{z}+\delta \boldsymbol{f}+O\left(\left\|\delta \phi^{t} \boldsymbol{z}\right\|^{2}\right) .
\end{aligned}
$$

Neglecting the higher-order term, we obtain, through Duhamel's principle,

$$
\delta \phi^{t} \boldsymbol{z}=\int_{0}^{t} \boldsymbol{T}_{\boldsymbol{z}}^{s} \mathrm{~d} s \delta \boldsymbol{f}
$$

where $\boldsymbol{T}_{\boldsymbol{z}}^{t}$ is the tangent map of (B.1), given by

$$
\frac{\partial}{\partial t} \boldsymbol{T}_{\boldsymbol{z}}^{t}=\nabla \boldsymbol{G}\left(\phi^{t} \boldsymbol{z}\right) \boldsymbol{T}_{\boldsymbol{z}}^{t}
$$

Then, by integrating over $\mu$ and taking $t \rightarrow \infty$, for the infinite time response of $\langle\boldsymbol{z}\rangle$ we obtain

$$
\delta\langle\boldsymbol{z}\rangle=\int_{0}^{\infty} \int_{\mathcal{A}} \boldsymbol{T}_{\boldsymbol{z}}^{s} \mathrm{~d} \mu(\boldsymbol{z}) \mathrm{d} s \delta \boldsymbol{f} .
$$

Assuming ergodicity of $\mu$ and replacing measure average with time average over a long-term trajectory $\boldsymbol{z}(t)$ of (B.1), we obtain

$$
\delta\langle\boldsymbol{z}\rangle=\int_{0}^{\infty}\left(\lim _{r \rightarrow \infty} \frac{1}{r} \int_{0}^{r} \boldsymbol{T}_{\boldsymbol{z}(\tau)}^{s} \mathrm{~d} \tau\right) \mathrm{d} s \delta \boldsymbol{f} .
$$

\section{Appendix C. Averaged dynamics with explicit time dependence.}

Here, we assume that the fast limiting system in (8.2), which includes both the $s$-dependent Wiener process $\boldsymbol{W}_{s}$ and the explicit $s$-dependence of $\boldsymbol{G}$ and $\boldsymbol{\sigma}$, can be expressed as a collection of the following dynamical systems: 
- A flow $\theta^{s}: \mathcal{Q} \rightarrow \mathcal{Q}$ on a measurable space $\mathcal{Q}$ with a distribution measure $\rho$, where $s$ is the time parameter. The elements $q \in \mathcal{Q}$ play the role of a bounded pseudo- "time" in the deterministic time-dependence of $\boldsymbol{F}, \boldsymbol{G}, \boldsymbol{\kappa}$, and $\boldsymbol{\sigma} . \theta^{s}$ has the following properties:

- The semigroup property: $\theta^{0}=I, \theta^{s} \theta^{r}=\theta^{s+r}$;

- $\theta$ is measure-preserving: $\rho(Q)=\rho\left(\theta^{s} Q\right)$ for any subset $Q \subset \mathcal{Q}$.

- A base flow $\vartheta^{s}: \Omega \rightarrow \Omega$, given over a probability space $\{\Omega, \mathcal{F}, \mathbb{P}\}$ (this probability space documents all realizations of the Wiener process $\left.\boldsymbol{W}_{s}(\omega)\right)$. The idea of this dynamical system is to "fast-forward" or "rewind" $\boldsymbol{W}_{s}(\omega)$ as needed by specifying the time parameter $s . \vartheta^{s}$ has the following properties:

- The semigroup property: $\vartheta^{0}=I, \vartheta^{s} \vartheta^{r}=\vartheta^{s+r}$;

- If $A \in \mathcal{F}$, then $\vartheta^{s} A$ is also in $\mathcal{F}$ for any $s$;

- Measure preservation: $\mathbb{P}(A)=\mathbb{P}\left(\vartheta^{s} A\right)$ for any $s$;

- The Wiener process $\boldsymbol{W}_{s}(\omega)$ satisfies $\boldsymbol{W}_{r}\left(\vartheta^{s} \omega\right)=\boldsymbol{W}_{s+r}(\omega)-\boldsymbol{W}_{s}(\omega)$.

- A $\theta, \vartheta$-cocycle $\phi_{\boldsymbol{x}, t}^{q, \omega, s}: \mathbb{R}^{N_{y}} \rightarrow \mathbb{R}^{N_{y}}$ with properties

$$
\phi_{\boldsymbol{x}, t}^{q, \omega, 0}=I, \quad \phi_{\boldsymbol{x}, t}^{q, \omega, s+r}=\phi_{\boldsymbol{x}, t}^{\theta^{r} q, \vartheta^{r} \omega, s} \phi_{\boldsymbol{x}, t}^{q, \omega, r}, \quad \forall(q, \omega) \in(\mathcal{Q}, \Omega),
$$

which represents a solution of the non-autonomous fast limiting system in (8.2).

Now, instead of considering a still compact global attractor $\mathcal{A}_{\boldsymbol{x}}$ for the autonomous dynamics like in Section 3 (which parametrically depends on the slow variables $\boldsymbol{x}$ ), here we consider a $q, \omega$-dependent family of compact global pullback-attracting sets $\mathcal{A}_{\boldsymbol{x}, t}(q, \omega) \subset \mathbb{R}^{N_{y}}$ which is $\theta, \vartheta$-invariant, that is,

$$
\phi_{\boldsymbol{x}, t}^{q, \omega, s} \mathcal{A}_{\boldsymbol{x}, t}(q, \omega)=\mathcal{A}_{\boldsymbol{x}, t}\left(\theta^{s} q, \vartheta^{s} \omega\right), \quad \lim _{s \rightarrow \infty} \operatorname{dist}\left(\mathcal{A}_{\boldsymbol{x}, t}(q, \omega), \phi_{\boldsymbol{x}, t}^{\theta^{-s} q, \vartheta^{-s} \omega, s} \mathcal{B}\right)=0,
$$

for all $q \in \mathcal{Q}, \mathbb{P}$-almost all $\omega \in \Omega$, and all compact subsets $\mathcal{B}$ of $\mathbb{R}^{N_{y}}$, where dist is the Hausdorff distance in $\mathbb{R}^{N_{y}}$. Then, the family of sets $\mathcal{A}_{\boldsymbol{x}, t}(q, \omega)$ is called the global pullback attractor of (8.2). Similarly, instead of a single invariant measure $\mu_{\boldsymbol{x}}$ one can consider a family of $\theta, \vartheta$-invariant measures $\mu_{\boldsymbol{x}, t}^{q, \omega}$ on $\mathcal{A}_{\boldsymbol{x}, t}(q, \omega)$ with the property

$$
\mu_{\boldsymbol{x}, t}^{q, \omega}\left(\phi_{\boldsymbol{x}, t}^{q, \omega, s} A\right)=\mu_{\boldsymbol{x}, t}^{\theta^{s} q, \vartheta^{s} \omega}(A), \quad \forall A \subset \mathcal{A}_{\boldsymbol{x}, t}(q, \omega) .
$$

At this point, let

$$
\begin{aligned}
\tilde{\boldsymbol{F}}\left(\boldsymbol{x}, \boldsymbol{z}, t, \theta^{s} q_{0}\right)=\boldsymbol{F}(\boldsymbol{x}, \boldsymbol{z}, t, s), & \tilde{\boldsymbol{G}}\left(\boldsymbol{x}, \boldsymbol{z}, t, \theta^{s} q_{0}\right)=\boldsymbol{G}(\boldsymbol{x}, \boldsymbol{z}, t, s), \\
\tilde{\boldsymbol{\kappa}}\left(\boldsymbol{x}, t, \theta^{s} q_{0}\right)=\boldsymbol{\kappa}(\boldsymbol{x}, t, s), & \tilde{\boldsymbol{\sigma}}\left(\boldsymbol{z}, t, \theta^{s} q_{0}\right)=\boldsymbol{\sigma}(\boldsymbol{z}, t, s),
\end{aligned}
$$

for all $\boldsymbol{x}, t, \boldsymbol{z}, s$, and some $q_{0} \in \mathcal{Q}$ which corresponds to the zero time $s=0$. Now, the slow limiting averaged system for (8.1) is given by

$$
\begin{gathered}
\mathrm{d} \boldsymbol{x}=\langle\boldsymbol{F}\rangle(\boldsymbol{x}, t) \mathrm{d} t+\langle\boldsymbol{\kappa}\rangle(\boldsymbol{x}, t) \mathrm{d} \boldsymbol{V}_{t}, \\
\langle\boldsymbol{F}\rangle(\boldsymbol{x}, t)=\int_{\mathcal{Q}} \int_{\Omega} \int_{\mathcal{A}_{\boldsymbol{x}, t}(q, \omega)} \tilde{\boldsymbol{F}}(\boldsymbol{x}, \boldsymbol{z}, t, q) \mathrm{d} \mu_{\boldsymbol{x}, t}^{q, \omega}(\boldsymbol{z}) \mathrm{d} \mathbb{P}(\omega) \mathrm{d} \rho(q), \\
\langle\boldsymbol{\kappa}\rangle(\boldsymbol{x}, t)\langle\boldsymbol{\kappa}\rangle(\boldsymbol{x}, t)^{T}=\int_{\mathcal{Q}} \tilde{\boldsymbol{\kappa}}(\boldsymbol{x}, t, q) \tilde{\boldsymbol{\kappa}}(\boldsymbol{x}, t, q)^{T} \mathrm{~d} \rho(q) \\
=\lim _{r \rightarrow \infty} \frac{1}{r} \int_{0}^{r} \boldsymbol{\kappa}(\boldsymbol{x}, t, s) \boldsymbol{\kappa}(\boldsymbol{x}, t, s)^{T} \mathrm{~d} s
\end{gathered}
$$


Here we assume that the cocycle in (C.1), which is a stochastic flow in $s$, is differentiable with respect to its initial condition [17], and therefore the corresponding equations for the averaged slow tangent map in (3.6) are given by

$$
\begin{array}{r}
\mathrm{d} \boldsymbol{T} \boldsymbol{X}_{\boldsymbol{x}}^{t_{0}, t}=\left[\left(\left\langle\frac{\partial \boldsymbol{F}}{\partial \boldsymbol{x}}\right\rangle(\boldsymbol{x}, t)+\boldsymbol{H}(\boldsymbol{x}, t)\right) \mathrm{d} t+\frac{\partial\langle\boldsymbol{\kappa}\rangle(\boldsymbol{x}, t)}{\partial \boldsymbol{x}} \mathrm{d} \boldsymbol{V}_{t}\right] \boldsymbol{T} \boldsymbol{X}_{\boldsymbol{x}}^{t_{0}, t}, \\
\left\langle\frac{\partial \boldsymbol{F}}{\partial \boldsymbol{x}}\right\rangle(\boldsymbol{x}, t)=\int_{\mathcal{Q}} \int_{\Omega} \int_{\mathcal{A}_{\boldsymbol{x}, t}(q, \omega)} \frac{\partial \tilde{\boldsymbol{F}}}{\partial \boldsymbol{x}}(\boldsymbol{x}, \boldsymbol{z}, t, q) \mathrm{d} \mu_{\boldsymbol{x}, t}^{q, \omega}(\boldsymbol{z}) \mathrm{d} \mathbb{P}(\omega) \mathrm{d} \rho(q), \\
\boldsymbol{H}(\boldsymbol{x}, t)=\int_{\mathcal{Q}} \int_{\Omega} \int_{\mathcal{A}_{\boldsymbol{x}, t}(q, \omega)} \tilde{\boldsymbol{F}}(\boldsymbol{x}, \boldsymbol{z}, t, q) \mathrm{d} \mu_{\boldsymbol{x}, t}^{\prime q, \omega}(\boldsymbol{z}) \mathrm{dP}(\omega) \mathrm{d} \rho(q),
\end{array}
$$

where $\mu^{\prime}$ is the derivative of $\mu$ with respect to the parameter $\boldsymbol{x}$. Observe that now the solution of (C.5), starting from a point $\boldsymbol{z}$, is given by the cocycle $\phi$ :

$$
\boldsymbol{z}(s)=\phi_{\boldsymbol{x}, t}^{q, \omega, s} \boldsymbol{z},
$$

where $q$ and $\omega$ denote parametric dependence on the starting time and probability space outcome for the Wiener process $\boldsymbol{W}_{s}(\omega)$, which means that $\phi$ satisfies

$$
\mathrm{d} \phi_{\boldsymbol{x}, t}^{q, \omega, s} \boldsymbol{z}=\tilde{\boldsymbol{G}}\left(\boldsymbol{x}, \phi_{\boldsymbol{x}, t}^{q, \omega} \boldsymbol{z}, t, \theta^{s} q\right) \mathrm{d} s+\tilde{\boldsymbol{\sigma}}\left(\phi_{\boldsymbol{x}, t}^{q, \omega, s} \boldsymbol{z}, t, \theta^{s} q\right) \mathrm{d} \boldsymbol{W}_{s}(\omega) .
$$

Now, let us denote the tangent map of the cocycle $\phi$ as

$$
\tilde{\boldsymbol{T}}_{\boldsymbol{x}, t, \boldsymbol{z}}^{q, \omega, s}=\frac{\partial}{\partial \boldsymbol{z}} \phi_{\boldsymbol{x}, t}^{q, \omega, s} \boldsymbol{z},
$$

which obviously obeys the chain rule

$$
\tilde{\boldsymbol{T}}_{\boldsymbol{x}, t, \boldsymbol{z}}^{q, \omega, s+r}=\tilde{\boldsymbol{T}}_{\boldsymbol{x}, t, \phi_{\boldsymbol{x}, t}^{\theta^{r} q, \vartheta^{r}} \boldsymbol{z}}^{q, \omega, s} \tilde{\boldsymbol{T}}_{\boldsymbol{x}, t, \boldsymbol{z}}^{q, \omega, r}, \quad \forall(q, \omega) \in(\mathcal{Q}, \Omega)
$$

and satisfies the equation

$$
\mathrm{d} \tilde{\boldsymbol{T}}_{\boldsymbol{x}, t, \boldsymbol{z}}^{q, \omega, s}=\left[\frac{\partial \tilde{\boldsymbol{G}}}{\partial \boldsymbol{z}}\left(\boldsymbol{x}, \phi_{\boldsymbol{x}, t}^{q, \omega, s} \boldsymbol{z}, t, \theta^{s} q\right) \mathrm{d} s+\frac{\partial \tilde{\boldsymbol{\sigma}}}{\partial \boldsymbol{z}}\left(\phi_{\boldsymbol{x}, t}^{q, \omega, s} \boldsymbol{z}, t, \theta^{s} q\right) \mathrm{d} \boldsymbol{W}_{s}(\omega)\right] \tilde{\boldsymbol{T}}_{\boldsymbol{x}, t, \boldsymbol{z}}^{q, \omega, s}
$$

Then, through Duhamel's principle, the $\boldsymbol{x}$-derivative of $\phi$ after elapsed time $a$ is computed as

$$
\frac{\partial}{\partial \boldsymbol{x}} \phi_{\boldsymbol{x}, t}^{q, \omega, a} \boldsymbol{z}=\int_{0}^{a} \tilde{\boldsymbol{T}}_{\boldsymbol{x}, t, \phi_{\boldsymbol{x}, t}^{q, w} \boldsymbol{z}}^{\theta^{s}, \vartheta^{s} \omega, a-s} \frac{\partial \tilde{\boldsymbol{G}}}{\partial \boldsymbol{x}}\left(\boldsymbol{x}, \phi_{\boldsymbol{x}, t}^{q, \omega, s} \boldsymbol{z}, t, \theta^{s} q\right) \mathrm{d} s
$$

while the average linear response of $\boldsymbol{F}$ to the changes in $\mu_{\boldsymbol{x}, t}^{q, \omega}$, which is after the elapsed time $a$ since $\boldsymbol{x}$ was changed, is given, in the pullback sense, as

$$
\begin{aligned}
\int_{\mathcal{A}_{\boldsymbol{x}, t}(q, \omega)} \tilde{\boldsymbol{F}}(\boldsymbol{x}, \boldsymbol{z}, t, q) \mathrm{d} \mu_{\boldsymbol{x}, t}^{\prime q, \omega}(\boldsymbol{z})=\int_{0}^{a} & \int_{\mathcal{A}_{\boldsymbol{x}, t}\left(\theta^{-a} q, \vartheta^{-a} \omega\right)} \frac{\partial \tilde{\boldsymbol{F}}}{\partial \boldsymbol{z}}(\boldsymbol{x}, \boldsymbol{z}, t, q) \tilde{\boldsymbol{T}}_{\boldsymbol{x}, t, \phi_{\boldsymbol{x}, t}^{q, \omega,-a} \boldsymbol{z}}^{\theta^{s-a} q, \vartheta^{s-a} \omega, a-s} \\
& \times \frac{\partial \tilde{\boldsymbol{G}}}{\partial \boldsymbol{x}}\left(\boldsymbol{x}, \phi_{\boldsymbol{x}, t}^{q, \omega, s-a} \boldsymbol{z}, t, \theta^{s-a} q\right) \mathrm{d} \mu_{\boldsymbol{x}, t}^{\theta^{-a} q, \vartheta^{-a} \omega}(\boldsymbol{z}) \mathrm{d} s .
\end{aligned}
$$


After applying (C.3), the above formula becomes

$$
\begin{aligned}
& \int_{\mathcal{A}_{\boldsymbol{x}, t}(q, \omega)} \tilde{\boldsymbol{F}}(\boldsymbol{x}, \boldsymbol{z}, t, q) \mathrm{d} \mu_{\boldsymbol{x}, t}^{\prime q, \omega}(\boldsymbol{z}) \\
&= \int_{0}^{a} \int_{\mathcal{A}_{\boldsymbol{x}, t}\left(\theta^{s-a} q, \vartheta^{s-a} \omega\right)}\left\{\frac{\partial \tilde{\boldsymbol{F}}}{\partial \boldsymbol{z}}\left(\boldsymbol{x}, \phi_{\boldsymbol{x}, t}^{\theta^{s-a} q, \theta^{s-a} \omega, a-s} \boldsymbol{z}, t, q\right)\right. \\
&\left.\times \tilde{\boldsymbol{T}}_{\boldsymbol{x}, t, \boldsymbol{z}}^{\theta^{s-a}, \vartheta^{s-a} \omega, a-s} \frac{\partial \tilde{\boldsymbol{G}}}{\partial \boldsymbol{x}}\left(\boldsymbol{x}, \boldsymbol{z}, t, \theta^{s-a} q\right)\right\} \mathrm{d} \mu_{\boldsymbol{x}, t}^{\theta^{s-a} q, \vartheta^{s-a} \omega}(\boldsymbol{z}) \mathrm{d} s(. \mathrm{C} .14)
\end{aligned}
$$

Averaging over $\rho$ and $\mathbb{P}$ yields

$$
\begin{gathered}
\int_{\mathcal{Q}} \int_{\Omega} \int_{\mathcal{A}_{\boldsymbol{x}, t}(q, \omega)} \tilde{\boldsymbol{F}}(\boldsymbol{x}, \boldsymbol{z}, t, q) \mathrm{d} \mu_{\boldsymbol{x}, t}^{\prime q, \omega}(\boldsymbol{z}) \mathrm{d} \mathbb{P}(\omega) \mathrm{d} \rho(q) \\
=\int_{0}^{a} \int_{\mathcal{Q}} \int_{\Omega} \int_{\mathcal{A}_{\boldsymbol{x}, t}\left(\theta^{s-a} q, \vartheta^{s-a} \omega\right)}\left\{\frac{\partial \tilde{\boldsymbol{F}}}{\partial \boldsymbol{z}}\left(\boldsymbol{x}, \phi_{\boldsymbol{x}, t}^{\theta^{s-a} q, \vartheta^{s-a} \omega, a-s} \boldsymbol{z}, t, q\right) \tilde{\boldsymbol{T}}_{\boldsymbol{x}, t, \boldsymbol{z}}^{\theta^{s-a}} q, \vartheta^{s-a} \omega, a-s\right. \\
\left.\times \frac{\partial \tilde{\boldsymbol{G}}}{\partial \boldsymbol{x}}\left(\boldsymbol{x}, \boldsymbol{z}, t, \theta^{s-a} q\right) \mathrm{d} \mu_{\boldsymbol{x}, t}^{\theta^{s-a} q, \vartheta^{s-a} \omega}(\boldsymbol{z})\right\} \mathrm{d} \mathbb{P}(\omega) \mathrm{d} \rho(q) \mathrm{d} s
\end{gathered}
$$

where, after using the invariance of $\rho$ and $\mathbb{P}$ under $\theta$ and $\vartheta$, respectively, and replacing $a-s$ with $s$ in the integral over $s$, we finally obtain

$$
\begin{gathered}
\int_{\mathcal{Q}} \int_{\Omega} \int_{\mathcal{A}_{\boldsymbol{x}, t}(q, \omega)} \tilde{\boldsymbol{F}}(\boldsymbol{x}, \boldsymbol{z}, t, q) \mathrm{d} \mu_{\boldsymbol{x}, t}^{q, \omega}(\boldsymbol{z}) \mathrm{d} \mathbb{P}(\omega) \mathrm{d} \rho(q) \\
=\int_{0}^{a} \int_{\mathcal{Q}} \int_{\Omega} \int_{\mathcal{A}_{\boldsymbol{x}, t}(q, \omega)}\left\{\frac{\partial}{\partial \boldsymbol{z}} \tilde{\boldsymbol{F}}\left(\boldsymbol{x}, \phi_{\boldsymbol{x}, t}^{q, \omega, s} \boldsymbol{z}, t, \theta^{s} q\right)\right. \\
\left.\times \tilde{\boldsymbol{T}}_{\boldsymbol{x}, t, \boldsymbol{z}}^{q, \omega} \frac{\partial}{\partial \boldsymbol{x}} \tilde{\boldsymbol{G}}(\boldsymbol{x}, \boldsymbol{z}, t, q)\right\} \mathrm{d} \mu_{\boldsymbol{x}, t}^{q, \omega}(\boldsymbol{z}) \mathrm{d} \mathbb{P}(\omega) \mathrm{d} \rho(q) \mathrm{d} s .
\end{gathered}
$$

Then the term $\boldsymbol{H}(\boldsymbol{x}, t)$ in (C.6) is formally given by sending the response time $a$ in the formula above to infinity:

$$
\begin{aligned}
\boldsymbol{H}(\boldsymbol{x}, t)=\int_{0}^{\infty} \int_{\mathcal{Q}} \int_{\Omega} \int_{\mathcal{A}_{\boldsymbol{x}, t}(q, \omega)} \frac{\partial}{\partial \boldsymbol{z}} \tilde{\boldsymbol{F}}\left(\boldsymbol{x}, \phi_{\boldsymbol{x}, t}^{q, \omega, s} \boldsymbol{z}, t, \theta^{s} q\right) \\
\times \tilde{\boldsymbol{T}}_{\boldsymbol{x}, t, \boldsymbol{z}, \boldsymbol{z}} \frac{\partial}{\partial \boldsymbol{x}} \tilde{\boldsymbol{G}}(\boldsymbol{x}, \boldsymbol{z}, t, q) \mathrm{d} \mu_{\boldsymbol{x}, t}^{q, \omega}(\boldsymbol{z}) \mathrm{d} \mathbb{P}(\omega) \mathrm{d} \rho(q) \mathrm{d} s .
\end{aligned}
$$

The assumption of the joint ergodicity of $\mathcal{Q}$ and $\Omega$ is that a sufficiently long trajectory $s$ completely samples $\mathcal{Q}$ and $\Omega$ through $\theta^{s}$ and $\vartheta^{s}$, respectively, with the corresponding statistical weights given by the product of the measures $\rho$ and $\mathbb{P}$. Under this assumption, one can replace the measure averages with the time averages, leading to (8.3). 


\section{REFERENCES}

[1] R. Abramov, Short-time linear response with reduced-rank tangent map, Chin. Ann. Math., 30B, 447-462, 2009.

[2] R. Abramov, Approximate linear response for slow variables of deterministic or stochastic dynamics with time scale separation, J. Comput. Phys., 229, 7739-7746, 2010.

[3] R. Abramov, Improved linear response for stochastically driven systems, Front. Math. China, accepted, 2011.

[4] R. Abramov and A. Majda, Quantifying uncertainty for non-Gaussian ensembles in complex systems, SIAM J. Sci. Comput., 26, 411-447, 2003.

[5] R. Abramov and A. Majda, Blended response algorithms for linear fluctuation-dissipation for complex nonlinear dynamical systems, Nonlin., 20, 2793-2821, 2007.

[6] R. Abramov and A. Majda, New approximations and tests of linear fluctuation-response for chaotic nonlinear forced-dissipative dynamical systems, J. Nonlin. Sci., 18, 303-341, 2008.

[7] R. Abramov and A. Majda, New algorithms for low frequency climate response, J. Atmos. Sci., $66,286-309,2009$.

[8] R. Abramov and A. Majda, Low frequency climate response of quasigeostrophic wind-driven ocean circulation, J. Phys. Oceanogr., accepted, 2011.

[9] T. Caraballo, G. Lukaszewicz, and J. Real, Pullback attractors for asymptotically compact non-autonomous dynamical systems, Nonlin. Anal., 64, 484-498, 2006.

[10] H. Crauel, A. Debussche, and F. Flandoli, Random attractors, J. Dyn. Diff. Eqs., 9, 307-341, 1997.

[11] D. Crommelin and E. Vanden-Eijnden, Subgrid scale parameterization with conditional Markov chains, J. Atmos. Sci., 65, 2661-2675, 2008.

[12] W. E, D. Liu, and E. Vanden-Eijnden, Analysis of multiscale methods for stochastic differential equations, Commun. Pure Appl. Math., 1544-1585, 2005.

[13] J. Eckmann and D. Ruelle, Ergodic theory of chaos and strange attractors, Rev. Mod. Phys., 57, 617-656, 1985.

[14] I. Fatkullin and E. Vanden-Eijnden, A computational strategy for multiscale systems with applications to Lorenz 96 model, J. Comput. Phys., 200, 605-638, 2004.

[15] C. Franzke, Dynamics of low-frequency variability: Barotropic mode, J. Atmos. Sci., 59, 29092897, 2002.

[16] M. Grote, A. Majda, and C. Grotta Ragazzo, Dynamic mean flow and small-scale interaction through topographic stress, J. Nonlin. Sci., 9, 89-130, 1999.

[17] H. Kunita, Stochastic Flows and Stochastic Differential Equations, Cambridge University Press, 1997.

[18] E. Lorenz, Predictability: A problem partly solved, in Proceedings of the Seminar on Predictability, Shinfield Park, Reading, England, 1996, ECMWF.

[19] E. Lorenz and K. Emanuel, Optimal sites for supplementary weather observations, J. Atmos. Sci., 55, 399-414, 1998.

[20] A. Majda, R. Abramov, and M. Grote, Information Theory and Stochastics for Multiscale Nonlinear Systems, CRM Monograph Series of Centre de Recherches Mathématiques, Université de Montréal, American Mathematical Society, 25, ISBN 0-8218-3843-1, 2005.

[21] A. Majda, I. Timofeyev, and E. Vanden-Eijnden, Models for stochastic climate prediction, Proc. Natl. Acad. Sci., 96, 14687-14691, 1999.

[22] A. Majda, I. Timofeyev, and E. Vanden-Eijnden, A mathematical framework for stochastic climate models, Commun. Pure Appl. Math., 54, 891-974, 2001.

[23] A. Majda, I. Timofeyev, and E. Vanden-Eijnden, Systematic strategies for stochastic mode reduction in climate, J. Atmos. Sci., 60, 1705-1722, 2003.

[24] G. Papanicolaou, Introduction to the asymptotic analysis of stochastic equations, in Modern Modeling of Continuum Phenomena, R. DiPrima ed., Lectures in Applied Mathematics, American Mathematical Society, 16, 1977.

[25] F. Risken, The Fokker-Planck Equation, Springer-Verlag, New York, 2nd ed., 1988.

[26] D. Ruelle, A measure associated with Axiom A attractors, Amer. J. Math., 98, 619-654, 1976.

[27] D. Ruelle, Chaotic Evolution and Strange Attractors, Cambridge University Press, 1989.

[28] D. Ruelle, General linear response formula in statistical mechanics, and the fluctuationdissipation theorem far from equilibrium, Phys. Lett. A, 245, 220-224, 1998.

[29] F. Selten, An efficient description of the dynamics of barotropic flow, J. Atmos. Sci., 52, 915936, 1995.

[30] G. Uhlenbeck and L. Ornstein, On the theory of the Brownian motion, Phys. Rev., 36, 823-841, 1930.

[31] E. Vanden-Eijnden, Numerical techniques for multiscale dynamical systems with stochastic 
effects, Commun. Math. Sci., 1, 385-391, 2003.

[32] V. Volosov, Averaging in systems of ordinary differential equations, Russian Math. Surveys, 17, 1-126, 1962.

[33] L.S. Young, What are SRB measures, and which dynamical systems have them? J. Stat. Phys., 108, 733-754, 2002. 